\begin{tabular}{|c|l|}
\hline Title & Hamilton-Jacobi equations with discontinuous source terms \\
\hline Author(s) & GIGA, Y OSHIKAZU; HA MA MUKI, NA O \\
\hline Citation & Hokkaido University Preprint Series in Mathematics, 987, 1-38 \\
\hline Issue Date & 2011-10-24 \\
\hline DOI & 10.14943/84134 \\
\hline Doc URL & http://hdl.handle.net/2115/69793 \\
\hline Type & bulletin (article) \\
\hline File Information & pre987.pdf \\
\hline
\end{tabular}

Instructions for use 


\title{
Hamilton-Jacobi equations with discontinuous source terms
}

\author{
Yoshikazu Giga $^{\mathrm{a}} \quad$ Nao Hamamuki ${ }^{\mathrm{b}}$ \\ ${ }^{a}$ Graduate School of Mathematical Sciences, University of Tokyo \\ 3-8-1 Komaba, Meguro-ku, Tokyo, 153-8914 Japan and \\ Department of Mathematics, Faculty of Sciences, King Abdulaziz University \\ P. O. Box 80203, Jeddah 21589, Saudi Arabia, labgiga@ms.u-tokyo.ac.jp \\ b Graduate School of Mathematical Sciences, University of Tokyo \\ 3-8-1 Komaba, Meguro-ku, Tokyo, 153-8914 Japan, hnao@ms.u-tokyo.ac.jp
}

\begin{abstract}
We study the initial-value problem for a Hamilton-Jacobi equation whose Hamiltonian is discontinuous with respect to state variables. Our motivation comes from a model describing the two dimensional nucleation in crystal growth phenomena. A typical equation has a semicontinuous source term. We introduce a new notion of viscosity solutions and prove among other results that the initial-value problem admits a unique global-in-time uniformly continuous solution for any bounded uniformly continuous initial data. We also give a representation formula of the solution as a value function by the optimal control theory with a semicontinuous running cost function.
\end{abstract}

Key words: viscosity solutions, Hamilton-Jacobi equations, discontinuous Hamiltonians

Mathematics Subject Classification: 35F21, 35F25, 49L25

\section{Introduction}

We consider the initial-value problem for the Hamilton-Jacobi equation of the form

$$
\text { (HJ) } \begin{cases}\partial_{t} u+H(x, \nabla u)=0 & \text { in } \mathbf{R}^{n} \times(0, T)=: Q, \\ \left.u\right|_{t=0}=u_{0} & \text { in } \mathbf{R}^{n}\end{cases}
$$

when the Hamiltonian $H$ is discontinuous in space variable $x \in \mathbf{R}^{n}$. Here $\nabla u$ denotes the spatial gradient, i.e., $\nabla u=\nabla_{x} u=\left(\partial_{x_{1}} u, \ldots, \partial_{x_{n}} u\right)$. A typical example we consider is the case when

$$
H(x, p)=-|p|-c I(x) \quad(c>0)
$$

with

$$
I(x):= \begin{cases}1 & (x=0) \\ 0 & (x \neq 0),\end{cases}
$$

where $|\cdot|$ stands for the standard Euclidean norm in $\mathbf{R}^{n}$. In other words, the source term can be discontinuous. Our main goal is to introduce a suitable definition of weak solution (by extending the theory of viscosity solutions) so that the initial-value problem admits a unique global-in-time solution for a general bounded Lipschitz continuous initial data $u_{0} \in B L i p\left(\mathbf{R}^{n}\right)$ or even just bounded uniformly continuous initial data $u_{0} \in B U C\left(\mathbf{R}^{n}\right)$.

Our motivation comes from crystal growth phenomena. One of key mechanisms of crystal growth is the two dimensional nucleation $([5,22])$. This growth is started by external supply of crystal molecules for a flat face. Such a source of supply is called a step source. The other mechanism is the spiral growth ([5]). According to [23], some high-temperature superconductor provides such a model and the authors proposed a macroscopic model including (1.1)-(1.3) approximating spiral growth on a crystal surface. Both situations can be modeled by Hamilton-Jacobi equations with discontinuous source terms if we interpret the phenomena in macroscopic point of view. 
Let us consider the typical case that there is a step source only at the origin and crystals grow at the uniform velocity 1 horizontally. Assume that the step source supplies crystal molecules at a rate of $c(>0)$ and let $u(x, t)$ be the height of crystals at position $x \in \mathbf{R}^{n}$ and time $t \in(0, T)$. (See Figure 1.) Then, the horizontal outward growth rate of crystals is given by $\partial_{t} u /|\nabla u|$. Since the horizontal growth

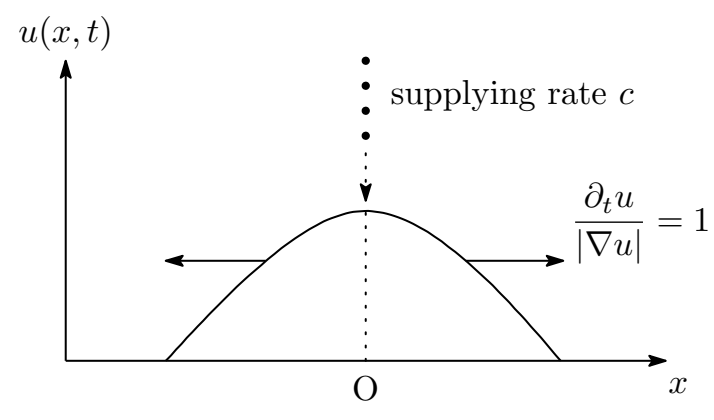

Figure 1: The step source at the origin.

speed is one, we have

$$
\partial_{t} u=|\nabla u|
$$

provided that $\nabla u \neq 0$. However, this equation does not include the effect of step sources. It is natural to postulate that the growth rate $\partial_{t} u$ at the origin should increase by $c$ due to the step source. The resulting equation is formally of the form

$$
\partial_{t} u-|\nabla u|=c I(x)
$$

The corresponding Hamiltonian (1.3) is not continuous but lower semicontinuous. The equation (1.4) is a Hamilton-Jacobi equation with a discontinuous source term. The physical intuition suggests that

$$
u^{c}(x, t)=c(t-|x|)_{+}
$$

is a solution for (1.4) when the initial-value equals zero (see Figure 2), where $a_{+}$denotes the positive part of $a \in \mathbf{R}$, i.e., $a_{+}=\max \{a, 0\}$. Such a "solution" is proposed in [19,23] by variational principle. The function $u^{c}$ is also obtained via approximation. More precisely, if we consider approximate Hamiltonians

$$
H^{\varepsilon}(x, p)=-|p|-c I^{\varepsilon}(x)
$$

with

$$
I^{\varepsilon}(x)=\left(1-\frac{|x|}{\varepsilon}\right)_{+}
$$

for $\varepsilon>0$ and solve (HJ) with $H^{\varepsilon}$ and $u_{0} \equiv 0$, it turns out that the unique viscosity solution of the approximate problem uniformly converges to $u^{c}$ as $\varepsilon \downarrow 0$. (See Example 3.2 for more details.) However, it is an important issue how to characterize $u^{c}$.

Unfortunately, we cannot expect the uniqueness of solutions for (1.4) even in Ishii's sense of viscosity solutions [18], where a discontinuous Hamiltonian is treated. Indeed, $u^{c}$ is a solution but $u^{\alpha}(x, t):=$ $\alpha(t-|x|)_{+}$for $\alpha \in[0, c]$ is also a solution with the zero initial data. This is caused by an inadequate effect of the discontinuous term. More precisely, in the standard definition of supersolutions we use the upper semicontinuous envelope of $H$, that is $H^{*}(x, p)=-|p|$, but then the term $c I(x)$ disappears which is a key term of our equation (1.4). Hence, in order to guarantee the uniqueness we must introduce some proper notion of supersolutions reflecting discontinuities and keep the notion of a subsolution in a standard way. Instead of using $H^{*}$ we are tempted to define a supersolution (D-supersolution) by requiring

$$
\tau+H(\hat{x}, p) \geqq 0 \quad \text { for all }(\hat{x}, \hat{t}) \in Q \text { and }(p, \tau) \in D^{-} u(\hat{x}, \hat{t}) \text {, }
$$



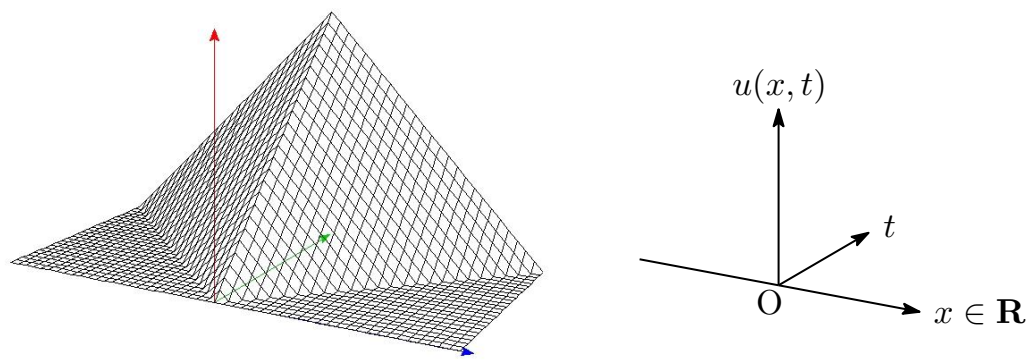

Figure 2: The intuitive solution of $\partial_{t} u-|\nabla u|=I(x), u_{0} \equiv 0$.

where $D^{-} u$ denotes the subdifferential. However, $u^{\alpha}$ is a $D$-supersolution no matter how $\alpha \in(0, c]$ is taken. We are led to introduce a notion of $\bar{D}$-supersolution by replacing $D^{-} u$ in (1.8) with $\bar{D}^{-} u$, a kind of "closure" of $D^{-} u$. We shall establish a general comparison principle for $\bar{D}$-super- and subsolutions. Applying this comparison principle we are able to establish a comparison principle for Lipschitz continuous $\bar{D}$-super- and subsolutions for (1.4). The reason we need Lipschitz continuity is that our general comparison principle needs continuity of $H$ in $x$ for large $|p|$ which excludes (1.4).

We next discuss the existence problem. Unfortunately, the intuitive solution $u^{c}$ in (1.5) is not a $\bar{D}$ supersolution. We have to weaken the definition of supersolutions by regarding the infimum of a family of $\bar{D}$-supersolutions as a "supersolution". We call such a supersolution an envelope supersolution. By definition we have a comparison principle for envelope super- and subsolutions. In this way, we introduce a notion of an envelope solution (envelope super- and subsolution) and construct a global-in-time solution by approximating equations with continuous Hamiltonians. It turns out that the envelope solution is a proper notion of the solution. Indeed, it is easy to see that $u^{c}$ is a unique envelope solution of (1.4) with the zero initial data. Moreover, we show that our solution preserves the Lipschitz continuity and uniform continuity of the initial data if Hamiltonian is coercive. Thus the envelope solution is unique for Lipschitz continuous initial data. Moreover, by a suitable approximation argument one is able to conclude that the envelope solution we constructed is unique even for bounded uniformly continuous initial data. The typical $H(x, p)$ we are concerned with is

$$
H(x, p)=H_{0}(x, p)-r(x),
$$

where $H_{0}$ is a continuous coercive Hamiltonian and $r$ is a bounded lower semicontinuous function. No convexity (concavity) assumption on $p \mapsto H(x, p)$ is imposed, though our example (1.3) is concave in $p$. In this case, we prove that there exists a unique uniformly continuous envelope solution for all bounded uniformly continuous initial data (Theorem 3.22).

The name "an envelope solution" was also introduced in [2] and [1] in order to deal with boundary conditions. They considered equations with continuous Hamiltonians, and defined the notion of envelope supersolutions as the infimum of standard viscosity supersolutions. Except on the boundary their envelope supersolution is a standard viscosity supersolution since the infimum of supersolutions in a domain is known to be a supersolution. Different from their solutions, our envelope solutions for discontinuous Hamiltonians may not be a $\bar{D}$-supersolution.

In the argument above we obtain the unique existence result for a Hamiltonian with the form (1.9) only when it is coercive. This is caused by a limitation of our comparison principle. In order to guarantee the continuity of $H(x, p)$ in $x$ for large $|p|$, we define a relaxed Hamiltonian $\hat{H}$ by regularizing the discontinuity of $H$. It turns out in several interesting examples that our envelope solution of (1.1) is also an envelope solution of (1.1) with a relaxed Hamiltonian $\hat{H}$ which permits a general comparison principle without assuming the Lipschitz continuity of solutions. Then, by regarding our envelope solution of the original problem as that of the relaxed problem, we establish the uniqueness of the envelope solutions but only for more restrictive Hamiltonians. Fortunately, this still applies the problem 
with finitely many source terms. (See (1.10) with (1.11).) It turns out that the relaxed Hamiltonian corresponding to $(1.3)$ is

$$
\hat{H}(x, p)=-|p|-(c I(x)-|p|)_{+},
$$

which is continuous if $|p| \geqq c$. (This Hamiltonian is coercive but very instructive to calculate $\hat{H}$.) Also, $u^{c}$ in (1.5) becomes an envelope solution of the relaxed problem.

If we consider the relaxed problem, there are more chance that an envelope solution is a $\bar{D}$-solution. We give its sufficient condition. Note that there still exists an envelope solution which is not a $\bar{D}$-solution.

Our theory applies to more physically interesting examples including

$$
\partial_{t} u-|\nabla u|=r(x),
$$

where $r: \mathbf{R}^{n} \rightarrow \mathbf{R}$ is bounded and upper semicontinuous, i.e., $r \in \operatorname{BUSC}\left(\mathbf{R}^{n}\right)$. A typical example in our mind is

$$
r(x)=\sum_{j=1}^{N} c_{j} I\left(x-a_{j}\right) \quad\left(c_{j}>0, a_{j} \in \mathbf{R}^{n}, a_{i} \neq a_{j}(i \neq j)\right) .
$$

This is the case that the step source is distributed at several singletons. It turns out that the resulting unique envelope solution with zero initial data is

$$
u(x, t)=\max _{j=1}^{N} c_{j}\left(t-\left|x-a_{j}\right|\right)_{+}
$$

which tells us that the envelope solution is the maximum of solutions for each step source. As an another example we have

$$
r(x)=c \chi_{S}(x) \quad\left(c>0 \text { and } S \text { is a nonempty closed subset of } \mathbf{R}^{n}\right) .
$$

Then (1.10) means that the step source is concentrated at a general set $S$. Here $\chi_{S}$ is the characteristic function of $S$, namely

$$
\chi_{S}(x):= \begin{cases}1 & (x \in S), \\ 0 & (x \notin S) .\end{cases}
$$

Our theory guarantees the unique existence of envelope solutions of (1.10) for general bounded uniformly continuous initial data. We are interested in establishing a representation formula of solutions based on the optimal control theory. However, the traditional method can be applied only for continuous equations. In this paper we adopt a discontinuous function appearing in our equation as a running cost function and prove that our envelope solution can be given via the value function of this discontinuous control problem under the some kind of controllability condition. Such an interpretation gives several explicit representation formulas of solutions. For example it guarantees that (1.12) is an envelope solution of (1.10) with (1.11) and $u_{0} \equiv 0$.

Our theory applies more general growth models including anisotropy. The typical form is

$$
\partial_{t} u-|\nabla u| U\left(\frac{-\nabla u}{|\nabla u|}\right) \sigma(x)=r(x) .
$$

Here $U(\mathbf{n}): S^{n-1}=\left\{x \in \mathbf{R}^{n}|| x \mid=1\right\} \rightarrow \mathbf{R}$ is the growth rate in the direction $\mathbf{n} \in S^{n-1}$ and $-\nabla u /|\nabla u|$ means the outward unit normal vector to the level sets of $u$. The function $\sigma: \mathbf{R}^{n} \rightarrow \mathbf{R}$ is called the surface supersaturation. Since $|p| U(-p /|p|) \rightarrow 0$ as $|p| \rightarrow 0$ provided that $U$ is continuous, (1.14) has no singularity contrary to its seemingly singular appearance. The unique existence result for (1.14) is included in Theorem 3.22 .

When the Hamiltonian is non-coercive $([23,26])$, the problem becomes more complicated. We cannot expect uniqueness results similar to the coercive cases. The difficulty may be seen from the following two examples. The first one is

$$
H(x, p)=-c I(x) \quad(c>0),
$$


which means that there is no horizontal growth. Obviously $u(x, t)=\operatorname{ct} I(x)$ seems to be the solution when the initial-value equals zero. However, the solution is not continuous and the uniqueness of solutions breaks down in our definition as will be mentioned in Example 3.16. The second one is

$$
H^{c}(x, p)=-\frac{|p|}{1+|p|}-c I(x) \quad(c>0) .
$$

This Hamiltonian arises in physical phenomena ([23]) where growth velocity is dependent on the gradient of the crystal surface. For $0<c<1$ we show that there exists a unique envelope solution for any bounded uniformly continuous initial data.

In [26] and [17] a step source is considered as a Dirichlet boundary condition. One may think that our envelope solution of (1.4) coincides with a solution of the Dirichlet boundary problem with $u(0, t)=c t+u_{0}(0)$ at the origin. This guess is correct provided that a slope of the initial data is less than or equal to $c$. However, if not, it turns out that the Dirichlet problem may give a different solution from our problem with (1.3). We also discuss a relation to the dynamic boundary condition $\partial_{t} u(0, t)=c$.

In this paper we mainly discuss the case when the given Hamiltonian $H(x, p)$ is lower semicontinuous with respect to $x$. We here recall some preceding studies about the viscosity solution theory for PDEs with discontinuous Hamiltonians. Shortly after the establishment of notions of viscosity solution, Ishii [18] studied discontinuous Hamiltonians with respect to the variables $t$ and $u$. Discontinuities in the space variable $x$ are investigated in many other works later.

For the stationary problem, the equation of eikonal type was studied by Newcomb and $\mathrm{Su}$ [20], Ostrov [21], Deckelnick and Elliott [10] and Soravia [25]. In [20] the authors considered the equation $H(\nabla u)=n(x)$. Here $H(p)$ is convex, coercive and positive except at $p=0$ and $n$ is assumed to be lower semicontinuous and positive. They introduced a suitable notion called Monge solutions, which, in the case of continuous Hamiltonians, are consistent with the usual viscosity solution. Briani and Davini [4] generalized the approach of Monge solutions for the equation $H(x, \nabla u)=0$, where $H(x, p)$ is only assumed to be Borel measurable and quasi-convex in $p$. Although we did not check, we expect that our envelope solution should agree with the Monge solution when the latter is available. The work by Soravia [24] is related to our results concerning the optimal control theory. The author of [24] considered the equation

$$
\lambda u(x)+\sup _{a \in A}\{-\langle f(x, a), \nabla u(x)\rangle-h(x, a)\}=g(x)
$$

with a Borel measurable function $g$. Here $\langle\cdot, \cdot\rangle$ denotes the standard Euclidean inner product. The author established a general uniqueness result in the sense of lower semicontinuous solutions, which was introduced by Barron and Jensen [3]. However, the uniqueness result does not apply to our setting since the definition of solutions like lower semicontinuous solutions is not suitable for (1.4). The reason is that it is impossible to choose the intuitive solution $u^{c}$ exclusively even if we impose an additional condition about test functions from the opposite side no matter which definition of solutions (standard, $D$ - or $\bar{D}$-) we use.

For the time-dependent problem, Camilli and Siconolfi [7] considered the equation $\partial_{t} u+H(x, \nabla u)=$ 0 , where the Hamiltonian $H(x, p)$ is measurable in $x$ and convex, coercive in $p$. The convexity is used to guarantee the Legendre transform and the equivalence of a.e. subsolution and viscosity subsolution. For discontinuity of different types, there are a few works on the equations of the form

$$
\partial_{t} u+f(x, t) h(x, \nabla u)=0
$$

with discontinuous $f$, which has important applications in front propagations. Deckelnick and Elliott [11] obtained the unique existence of continuous viscosity solutions for the one space dimensional case when $f(x, t)=a(x)$ and $h(x, p)=\sqrt{1+p^{2}}$, where $a$ is assumed to be bounded, of bounded variation and one-sided Lipschitz continuous. Afterwards Chen and $\mathrm{Hu}$ [8] studied a more general case when $f$ depends on $t$ but $h$ depends only on $p$. They assumed that $f$ is positive, bounded and measurable and $h$ is non-negative and Lipschitz continuous. More recently, with the optimal control theory involved De Zan and Soravia [12] discussed the unique existence of solutions when $h$ depends also on $x$ while $f$ is independent of $t$ and piecewise Lipschitz continuous across Lipschitz hypersurfaces. Our results are therefore different from these above. The discontinuity of Hamiltonians we are concerned with is given as a source term instead of the jump of propagating speed, which is also studied recently in [16]. 
For the second order equation, Caffarelli, Crandall, Kocan and Swięch [6] studied fully nonlinear and uniformly elliptic PDEs by utilizing $L^{p}$-viscosity solution theory. However, the situation is quite different from ours.

This paper is organized as follows. In Section 2 we first define some notions of solutions. Then we establish two types of comparison principles for $\bar{D}$-solutions; a general version which excludes (1.4) and a Lipschitz version which includes (1.4) but needs Lipschitz continuity of solutions. Section 3 is devoted to existence problems of solutions. We prove that there exists a unique envelope solution of (HJ) when $H$ is coercive. Section 4 deals with relaxed Hamiltonians. After introducing the relaxed Hamiltonians, we deduce a unique existence result of envelope solutions without the coercivity assumption. Also, we discuss the existence of $\bar{D}$-solutions. Section 5 is dedicated to showing some examples of envelope solutions. We also mention the relation between our envelope solutions and solutions of Dirichlet boundary problems.

\section{Proper definition of solutions and comparison principles}

\subsection{Definition of solutions}

We first recall the notion of super- and subdifferentials to define a viscosity solution. For $u: Q \rightarrow \mathbf{R}$ and $(\hat{x}, \hat{t}) \in Q$ we set a superdifferential $D_{Q}^{+} u(\hat{x}, \hat{t})$ and an extended superdifferential $\bar{D}_{Q}^{+} u(\hat{x}, \hat{t})$ by

$$
\begin{aligned}
& D_{Q}^{+} u(\hat{x}, \hat{t}) \\
:= & \left\{(p, \tau) \in \mathbf{R}^{n} \times \mathbf{R} \mid \exists \phi \in C^{1}(Q), \max _{Q}(u-\phi)=(u-\phi)(\hat{x}, \hat{t}), \quad(p, \tau)=\left(\nabla \phi, \partial_{t} \phi\right)(\hat{x}, \hat{t})\right\}, \\
& \bar{D}_{Q}^{+} u(\hat{x}, \hat{t}) \\
:= & \left\{\begin{array}{l|c}
(p, \tau) \in \mathbf{R}^{n} \times \mathbf{R} \mid \begin{array}{c}
\exists\left\{\left(x_{m}, t_{m}\right)\right\}_{m \in \mathbf{N}} \subset Q, \exists\left\{\left(p_{m}, \tau_{m}\right)\right\}_{m \in \mathbf{N}} \subset \mathbf{R}^{n} \times \mathbf{R}, \\
\operatorname{such} \text { that }\left(p_{m}, \tau_{m}\right) \in D_{Q}^{+} u\left(x_{m}, t_{m}\right),\left(x_{m}, t_{m}\right) \rightarrow(\hat{x}, \hat{t}), \\
\left(p_{m}, \tau_{m}\right) \rightarrow(p, \tau), u\left(x_{m}, t_{m}\right) \rightarrow u(\hat{x}, \hat{t}) \text { as } m \rightarrow \infty
\end{array}
\end{array}\right\},
\end{aligned}
$$

where $\mathbf{N}:=\{1,2,3, \ldots\}$. We denote a subdifferential $D_{Q}^{-} u(\hat{x}, \hat{t})$ and an extended subdifferential $\bar{D}_{Q}^{-} u(\hat{x}, \hat{t})$ by $D_{Q}^{-} u(\hat{x}, \hat{t}):=-D_{Q}^{+}(-u)(\hat{x}, \hat{t})$ and $\bar{D}_{Q}^{-} u(\hat{x}, \hat{t}):=-\bar{D}_{Q}^{+}(-u)(\hat{x}, \hat{t})$. We can also define $D_{Q}^{-}$and $\bar{D}_{Q}^{-}$by replacing, respectively, max by min in (2.1) and $D_{Q}^{+}$by $D_{Q}^{-}$in (2.2). Index $Q$ is often omitted. It is known that $D^{+}$and $D^{-}$are closed convex subset of $\mathbf{R}^{n} \times \mathbf{R}$. (See [2, Lemma II.1.8.(a)].)

We call $\phi \in C^{1}(Q)$ a corresponding test function for $(p, \tau) \in D^{+} u(\hat{x}, \hat{t})$, where $\phi$ appears in (2.1). One can take such $\phi$ as a separated form, i.e., $\phi(x, t)=\psi(x)+g(t)$ with $\psi \in C^{1}\left(\mathbf{R}^{n}\right)$ and $g \in C^{1}(0, T)$. (See [15, Proposition 2.2.3.(i)].) Moreover we call $\left\{\left(x_{m}, t_{m}\right),\left(p_{m}, \tau_{m}\right)\right\}_{m \in \mathbf{N}} \subset Q \times D^{+} u\left(x_{m}, t_{m}\right)$ a defining approximate sequence for $(p, \tau) \in \bar{D}^{+} u(\hat{x}, \hat{t})$, where $\left(x_{m}, t_{m}\right)$ and $\left(p_{m}, \tau_{m}\right)$ are given in $(2.2)$.

Definition 2.1. Let $H=H(x, p)$ be a real valued function defined in $\mathbf{R}^{n} \times \mathbf{R}^{n}$ and let $u$ be a real valued function in $Q$.

(1) We call u a (standard) viscosity supersolution (resp. subsolution) of (1.1) if $u$ is bounded from below (resp. from above) in $Q$ and

$$
\begin{aligned}
\tau+H^{*}(\hat{x}, p) \geqq 0 & \text { for all }(\hat{x}, \hat{t}) \in Q \text { and }(p, \tau) \in D_{Q}^{-} u_{*}(\hat{x}, \hat{t}) . \\
\left(\text { resp. } \tau+H_{*}(\hat{x}, p) \leqq 0\right. & \text { for all } \left.(\hat{x}, \hat{t}) \in Q \text { and }(p, \tau) \in D_{Q}^{+} u_{*}(\hat{x}, \hat{t}) .\right)
\end{aligned}
$$

We denote by $S U P(H)$ and $S U B(H)$ respectively the set of all supersolutions and subsolutions of (1.1).

(2) If $u \in S U P(H)$ (resp. $u \in S U B(H)$ ) defined in $Q_{0}:=\mathbf{R}^{n} \times[0, T)$ is continuous on $\mathbf{R}^{n} \times\{0\}$ and satisfies the initial condition (1.2), it is called a viscosity supersolution (resp. subsolution) of (HJ) and then we write $u \in S U P\left(H, u_{0}\right)$ (resp. $u \in S U B\left(H, u_{0}\right)$ ). 
(3) We say that $u$ is a viscosity solution if it is both a viscosity supersolution and a viscosity subsolution. Define $S O L(H):=S U P(H) \cap S U B(H)$ and $S O L\left(H, u_{0}\right):=S U P\left(H, u_{0}\right) \cap S U B\left(H, u_{0}\right)$.

Remark 2.2. (1) For any subset $L \subset \mathbf{R}^{N}$ and $h: L \rightarrow \mathbf{R}$ we denote the upper semicontinuous envelope (resp. lower semicontinuous envelope) by $h^{*}$ (resp. $\left.h_{*}\right): \bar{L} \rightarrow \mathbf{R} \cup\{ \pm \infty\}$, which is as follows:

$$
\begin{aligned}
h^{*}(z) & :=\limsup _{y \rightarrow z} h(y)=\lim _{\delta \downarrow 0} \sup \left\{h(y) \mid y \in \bar{B}_{\delta}(z) \cap L\right\} \\
\text { resp. } h_{*}(z) & \left.:=\liminf _{y \rightarrow z} h(y)=\liminf _{\delta \downarrow 0}\left\{h(y) \mid y \in \bar{B}_{\delta}(z) \cap L\right\}\right) \quad(z \in \bar{L}),
\end{aligned}
$$

where $\bar{B}_{r}(x)$ stands for the closed ball with center $x$ and radius $r$. (We denote the open ball by $B_{r}(x)$.) The function $h^{*}$ is characterized as the smallest upper semicontiuous function on $\bar{L}$ that is greater than $h$ on $L$, while $h_{*}$ is the greatest lower semicontiuous function on $\bar{L}$ that is smaller than $h$ on $L$.

(2) We can replace $D_{Q}^{-}$in $(2.3)$ by $\bar{D}_{Q}^{-}$since $H^{*}$ is applied in the definition. This can be easily shown by taking limits.

(3) It often assume one side local boundedness of sub- and supersolutions instead of global boundedness in the literature. We impose the boundedness assumption to simplify the argument. Also, when we think of the initial value problem $(\mathrm{HJ})$, we require solutions to be continuous at $t=0$ for the sake of simplicity.

Example 2.3. Let us consider (HJ) with (1.3) and $u_{0} \equiv 0$. It is easy to verify that functions $u^{\alpha}(x, t):=$ $\alpha(t-|x|)_{+}(0 \leqq \alpha \leqq c)$ are all viscosity solutions of this initial-value problem in the standard sense above. We must therefore strengthen the definition of solutions in order to get uniqueness. As mentioned in Section 1, we adopt a new definition in which $H$ instead of $H^{*}$ is used in (2.3). However, notice that in this case the definition by $D_{Q}^{-}$and that by $\bar{D}_{Q}^{-}$are different.

Definition 2.4. Let $u: Q \rightarrow \mathbf{R}$. We call $u$ a $D$-viscosity supersolution (resp. $\bar{D}$-viscosity supersolution) of (1.1) if $u$ is bounded from below in $Q$ and

$$
\begin{array}{r}
\tau+H(\hat{x}, p) \geqq 0 \quad \text { for all }(\hat{x}, \hat{t}) \in Q \text { and }(p, \tau) \in D_{Q}^{-} u_{*}(\hat{x}, \hat{t}) . \\
\left(\text { resp. } \tau+H(\hat{x}, p) \geqq 0 \quad \text { for all }(\hat{x}, \hat{t}) \in Q \text { and }(p, \tau) \in \bar{D}_{Q}^{-} u_{*}(\hat{x}, \hat{t}) .\right)
\end{array}
$$

We denote by D-SUP $(H)$ (resp. $\bar{D}$-SUP $(H)$ ) a set which consists of all D-supersolutions (resp. $\bar{D}$-supersolutions) of (1.1). Moreover we similarly define a corresponding viscosity subsolution, solution, solution of the initial-value problem, and set notations by marking $D$ - or $\bar{D}$-.

About three notions of supersolutions defined so far we have the inclusion relation $S U P(H) \supset$ $D-S U P(H) \supset \bar{D}-S U P(H)$ in general, and these sets are the same for upper semicontinuous $H$. Since we mainly think of a lower continuous $H$, as for subsolutions we have $S U B(H)=D-S U B(H)=\bar{D}-S U B(H)$ in many cases.

Example 2.5. We revisit the Example 2.3. How about solutions of our equation in the sense of $D$ or $\bar{D}$-solutions? Since there is no smooth function that touches $u^{\alpha}$ from below at $(0, \hat{t})(\hat{t}>0)$ when $\alpha \in(0, c]$, they all become $D$-solutions. (When $\alpha=0$, the function $u \equiv 0$ is not a $D$-supersolution.) This suggests that $D$-solutions are still not unique. For this reason we adopt $\bar{D}$-solutions as a proper definition for the moment, and we will show comparison principles for such solutions in the next subsection.

\subsection{Comparison principles}

We will show comparison principles (CP for short), which are important to prove uniqueness of solutions. The following two assumptions are standard for usual CP.

$\left(\mathrm{H}_{p}\right)$ There exists a modulus $\omega_{1} \in \mathcal{M}$ such that $|H(x, p)-H(x, q)| \leqq \omega_{1}(|p-q|)$ for all $x, p, q \in \mathbf{R}^{n}$. 
$\left(\mathrm{H}_{x}\right)$ There exists a modulus $\omega_{2} \in \mathcal{M}$ such that $|H(x, p)-H(y, p)| \leqq \omega_{2}((1+|p|)|x-y|)$ for all $x, y, p \in \mathbf{R}^{n}$.

Here we denote by $\mathcal{M}$ the set of all moduli of continuity, namely

$$
\mathcal{M}:=\{\omega:[0, \infty) \rightarrow[0, \infty) \mid \omega(0)=0, \omega \text { is continuous at } 0 \text { and nondecreasing on }[0, \infty) .\} .
$$

We still use $\left(\mathrm{H}_{p}\right)$ now. Since we should treat discontinuous Hamiltonians with respect to the space variable, we weaken $\left(\mathrm{H}_{x}\right)$ in the following manner.

$\left(\mathrm{H}_{x N}\right)$ There exist a modulus $\omega_{2} \in \mathcal{M}$ and a constant $N>0$ such that $|H(x, p)-H(y, p)| \leqq \omega_{2}((1+$ $|p|)|x-y|)$ for all $x, y \in \mathbf{R}^{n}$ and $p \in \mathbf{R}^{n} \backslash B_{N}(0)$.

This condition means that $\left(\mathrm{H}_{x}\right)$ holds if $|p|$ is large. Note that (1.3) does not satisfy $\left(\mathrm{H}_{x N}\right)$.

Before stating our CP, we check that $H$ satisfying $\left(\mathrm{H}_{p}\right)$ and $\left(\mathrm{H}_{x N}\right)$ is locally bounded in $\mathbf{R}^{n} \times \mathbf{R}^{n}$. This fact will be used in the proof of CP. Since the local boundedness is clear in $\mathbf{R}^{n} \times\left(\mathbf{R}^{n} \backslash \bar{B}_{N}(0)\right)$, we show that $H$ is bounded in $\bar{B}_{1}(x) \times \bar{B}_{1}(p)$ for any $(x, p) \in \mathbf{R}^{n} \times \bar{B}_{N}(0)$. Take any $(y, q) \in \bar{B}_{1}(x) \times \bar{B}_{1}(p)$ and $p^{\prime} \in \mathbf{R}^{n}$ such that $N \leqq\left|p^{\prime}\right| \leqq N+1$. Then, we calculate

$$
\begin{aligned}
|H(x, p)-H(y, q)| & \leqq\left|H(x, p)-H\left(x, p^{\prime}\right)\right|+\left|H\left(x, p^{\prime}\right)-H\left(y, p^{\prime}\right)\right|+\left|H\left(y, p^{\prime}\right)-H(y, q)\right| \\
& \leqq 2 \omega_{1}\left(\left|p-p^{\prime}\right|+1\right)+\omega_{2}\left(\left(1+\left|p^{\prime}\right|\right)|x-y|\right) \\
& \leqq 2 \omega_{1}(2 N+2)+\omega_{2}(N+2),
\end{aligned}
$$

which yields our claim.

Theorem 2.6 (CP-general version). Assume that $H$ satisfies $\left(\mathrm{H}_{p}\right)$ and $\left(\mathrm{H}_{x N}\right)$. Let $u$ and $v: Q_{0} \rightarrow \mathbf{R}$ be, respectively, bounded from above and bounded from below in $Q_{0}$. Assume that $u \in \bar{D}-S U B(H)$ and $v \in \bar{D}$-SUP $(H)$. If $u^{*}(\cdot, 0) \leqq v_{*}(\cdot, 0)$ in $\mathbf{R}^{n}$, then $u^{*} \leqq v_{*}$ in $Q$.

Though our assumption for $H$ is weaker than the classical one, our definition of solutions is stronger, and so we can keep balance.

Proof. 1. Suppose by contradiction that there would exist $\left(x_{0}, t_{0}\right) \in \mathbf{R}^{n} \times(0, T)$ such that $u^{*}\left(x_{0}, t_{0}\right)-$ $v_{*}\left(x_{0}, t_{0}\right)=: A>0$. We define a function $\mathcal{F}:\left(\mathbf{R}^{n} \times[0, T]\right)^{2} \rightarrow \mathbf{R} \cup\{-\infty\}$ by

$$
\begin{aligned}
\mathcal{F}(x, t, y, s) & :=u^{*}(x, t)-v_{*}(y, s)-\Psi(x, t, y, s) \\
\text { with } \Psi(x, t, y, s) & :=\frac{1}{2 \varepsilon^{2}}\left(|x-y|^{2}+|t-s|^{2}\right)+\beta f(x)+\frac{\alpha}{T-t},
\end{aligned}
$$

where $\alpha \in\left(0, A\left(T-t_{0}\right)\right), \beta>0, \varepsilon>0$ and $f(x)=\sqrt{1+\left|x-x_{0}\right|^{2}}-1$. Note that $f \geqq 0, f \in C^{1}\left(\mathbf{R}^{n}\right)$ and $|\nabla f| \leqq 1$. Also, by the choice of $\alpha$, we have

$$
\mathcal{F}\left(x_{0}, t_{0}, x_{0}, t_{0}\right)=u^{*}\left(x_{0}, t_{0}\right)-v_{*}\left(x_{0}, t_{0}\right)-\frac{\alpha}{T-t_{0}}>0 .
$$

Since $u$ and $-v$ are bounded from above, $\mathcal{F}$ attains its maximum in $\left(\mathbf{R}^{n} \times[0, T]\right)^{2}$ at some $\left(x^{\varepsilon}, t^{\varepsilon}, y^{\varepsilon}, s^{\varepsilon}\right) \in$ $\left(\mathbf{R}^{n} \times[0, T)\right)^{2}$. Then, we see

$$
\mathcal{F}\left(x^{\varepsilon}, t^{\varepsilon}, y^{\varepsilon}, s^{\varepsilon}\right) \geqq \mathcal{F}\left(x_{0}, t_{0}, x_{0}, t_{0}\right)>0
$$

2. Set $M:=\sup _{Q_{0}} u^{*}+\sup _{Q_{0}}\left(-v_{*}\right)(<\infty)$. Then we have $\beta f\left(x^{\varepsilon}\right)<M$ by $(2.4)$, and hence $\left\{x^{\varepsilon}\right\}_{\varepsilon>0}$ is bounded. Furthermore, since we also have $\left|x^{\varepsilon}-y^{\varepsilon}\right| \leqq \sqrt{2 M} \varepsilon$ and $\left|t^{\varepsilon}-s^{\varepsilon}\right| \leqq \sqrt{2 M} \varepsilon$ by (2.4), we may assume that there exists some $(\hat{x}, \hat{t}) \in \mathbf{R}^{n} \times[0, T]$ such that

$$
\lim _{\varepsilon \downarrow 0}\left(x^{\varepsilon}, t^{\varepsilon}, y^{\varepsilon}, s^{\varepsilon}\right)=(\hat{x}, \hat{t}, \hat{x}, \hat{t}) .
$$

Here, we claim that $\hat{t} \in(0, T)$. By (2.4) we observe

$$
0<\limsup _{\varepsilon \downarrow 0} \mathcal{F}\left(x^{\varepsilon}, t^{\varepsilon}, y^{\varepsilon}, s^{\varepsilon}\right) \leqq \mathcal{F}(\hat{x}, \hat{t}, \hat{x}, \hat{t}) .
$$


However, since we have

$$
\begin{aligned}
\mathcal{F}(\hat{x}, 0, \hat{x}, 0) & =u^{*}(\hat{x}, 0)-v_{*}(\hat{x}, 0)-\beta f(\hat{x})-\frac{\alpha}{T}<0, \\
\mathcal{F}(\hat{x}, T, \hat{x}, T) & =-\infty
\end{aligned}
$$

it follows that $\hat{t} \neq 0$ and $\hat{t} \neq T$.

3. We remark that

$$
\mathcal{F}(\hat{x}, \hat{t}, \hat{x}, \hat{t}) \leqq \mathcal{F}\left(x^{\varepsilon}, t^{\varepsilon}, y^{\varepsilon}, s^{\varepsilon}\right)
$$

because $\left(x^{\varepsilon}, t^{\varepsilon}, y^{\varepsilon}, s^{\varepsilon}\right)$ is the maximizer of $\mathcal{F}$. In view of (2.6) we calculate

$$
\begin{aligned}
& \limsup _{\varepsilon \downarrow 0} \frac{1}{2 \varepsilon^{2}}\left(\left|x^{\varepsilon}-y^{\varepsilon}\right|^{2}+\left|t^{\varepsilon}-s^{\varepsilon}\right|^{2}\right) \\
\leqq & \limsup _{\varepsilon \downarrow 0}\left\{-\mathcal{F}(\hat{x}, \hat{t}, \hat{x}, \hat{t})+u^{*}\left(x^{\varepsilon}, t^{\varepsilon}\right)-v_{*}\left(y^{\varepsilon}, s^{\varepsilon}\right)-\beta f\left(x^{\varepsilon}\right)-\frac{\alpha}{T-t^{\varepsilon}}\right\} \\
\leqq & -\mathcal{F}(\hat{x}, \hat{t}, \hat{x}, \hat{t})+u^{*}(\hat{x}, \hat{t})-v_{*}(\hat{x}, \hat{t})-\beta f(\hat{x})-\frac{\alpha}{T-\hat{t}}=0 .
\end{aligned}
$$

Hence

$$
\lim _{\varepsilon \downarrow 0} \frac{\left|x^{\varepsilon}-y^{\varepsilon}\right|}{\varepsilon}=0, \quad \lim _{\varepsilon \downarrow 0} \frac{\left|t^{\varepsilon}-s^{\varepsilon}\right|}{\varepsilon}=0 .
$$

Also, by (2.6) and the upper semicontinuity of $\mathcal{F}$, we observe

$$
\mathcal{F}(\hat{x}, \hat{t}, \hat{x}, \hat{t}) \leqq \liminf _{\varepsilon \downarrow 0} \mathcal{F}\left(x^{\varepsilon}, t^{\varepsilon}, y^{\varepsilon}, s^{\varepsilon}\right) \leqq \limsup _{\varepsilon \downarrow 0} \mathcal{F}\left(x^{\varepsilon}, t^{\varepsilon}, y^{\varepsilon}, s^{\varepsilon}\right) \leqq \mathcal{F}(\hat{x}, \hat{t}, \hat{x}, \hat{t}),
$$

which means

$$
\lim _{\varepsilon \downarrow 0} \mathcal{F}\left(x^{\varepsilon}, t^{\varepsilon}, y^{\varepsilon}, s^{\varepsilon}\right)=\mathcal{F}(\hat{x}, \hat{t}, \hat{x}, \hat{t})
$$

This equality and (2.7) implies

$$
\lim _{\varepsilon \downarrow 0}\left\{u^{*}\left(x^{\varepsilon}, t^{\varepsilon}\right)-v_{*}\left(y^{\varepsilon}, s^{\varepsilon}\right)\right\}=u^{*}(\hat{x}, \hat{t})-v_{*}(\hat{x}, \hat{t}) .
$$

Now, we also observe

$$
\begin{aligned}
u^{*}(\hat{x}, \hat{t}) & \geqq \limsup _{\varepsilon \downarrow 0} u^{*}\left(x^{\varepsilon}, t^{\varepsilon}\right) \geqq \liminf _{\varepsilon \downarrow 0} u^{*}\left(x^{\varepsilon}, t^{\varepsilon}\right) \\
& =\liminf _{\varepsilon \downarrow 0}\left\{\left(u^{*}\left(x^{\varepsilon}, t^{\varepsilon}\right)-v_{*}\left(y^{\varepsilon}, s^{\varepsilon}\right)\right)+v_{*}\left(y^{\varepsilon}, s^{\varepsilon}\right)\right\} \\
& \geqq\left(u^{*}(\hat{x}, \hat{t})-v_{*}(\hat{x}, \hat{t})\right)+v_{*}(\hat{x}, \hat{t})=u^{*}(\hat{x}, \hat{t}) .
\end{aligned}
$$

Consequently it follows that

$$
\lim _{\varepsilon \downarrow 0} u^{*}\left(x^{\varepsilon}, t^{\varepsilon}\right)=u^{*}(\hat{x}, \hat{t}), \quad \lim _{\varepsilon \downarrow 0} v_{*}\left(y^{\varepsilon}, s^{\varepsilon}\right)=v_{*}(\hat{x}, \hat{t}) .
$$

4. We next calculate the first derivatives of $\Psi$ at $\left(x^{\varepsilon}, t^{\varepsilon}, y^{\varepsilon}, s^{\varepsilon}\right)$.

$$
\begin{aligned}
p_{x}^{\varepsilon} & :=\nabla_{x} \Psi\left(x^{\varepsilon}, t^{\varepsilon}, y^{\varepsilon}, s^{\varepsilon}\right)=\frac{1}{\varepsilon^{2}}\left(x^{\varepsilon}-y^{\varepsilon}\right)+\beta \nabla f\left(x^{\varepsilon}\right), \\
p_{y}^{\varepsilon} & :=\nabla_{y} \Psi\left(x^{\varepsilon}, t^{\varepsilon}, y^{\varepsilon}, s^{\varepsilon}\right)=-\frac{1}{\varepsilon^{2}}\left(x^{\varepsilon}-y^{\varepsilon}\right) \\
\tau^{\varepsilon} & :=\partial_{t} \Psi\left(x^{\varepsilon}, t^{\varepsilon}, y^{\varepsilon}, s^{\varepsilon}\right)=\frac{1}{\varepsilon^{2}}\left(t^{\varepsilon}-s^{\varepsilon}\right)+\frac{\alpha}{\left(T-t^{\varepsilon}\right)^{2}} \\
\sigma^{\varepsilon} & :=\partial_{s} \Psi\left(x^{\varepsilon}, t^{\varepsilon}, y^{\varepsilon}, s^{\varepsilon}\right)=-\frac{1}{\varepsilon^{2}}\left(t^{\varepsilon}-s^{\varepsilon}\right) .
\end{aligned}
$$


By the definitions of $D^{ \pm}$we have

$$
\left\{\begin{array}{l}
\left(p_{x}^{\varepsilon}, \tau^{\varepsilon}\right) \in D^{+} u^{*}\left(x^{\varepsilon}, t^{\varepsilon}\right), \\
\left(-p_{y}^{\varepsilon},-\sigma^{\varepsilon}\right) \in D^{-} v_{*}\left(y^{\varepsilon}, s^{\varepsilon}\right),
\end{array}\right.
$$

and therefore

$$
\left\{\begin{array}{l}
\tau^{\varepsilon}+H\left(x^{\varepsilon}, p_{x}^{\varepsilon}\right) \leqq 0, \\
-\sigma^{\varepsilon}+H\left(y^{\varepsilon},-p_{y}^{\varepsilon}\right) \geqq 0
\end{array}\right.
$$

since $u \in D$-SUB $(H)$ and $v \in D$-SUP $(H)$.

Here we discuss two different cases for subsequences of $\left\{p_{y}^{\varepsilon}\right\}_{\varepsilon>0}$ :

There exists a sequence $\{\varepsilon(j)\}_{j \in \mathbf{N}}$ such that $\varepsilon(j) \downarrow 0(j \rightarrow \infty)$ and

Case 1: $\left|p_{y}^{\varepsilon(j)}\right| \rightarrow \infty$.

Case 2: $p_{y}^{\varepsilon(j)} \rightarrow-\bar{p}$ for some $\bar{p} \in \mathbf{R}^{n}$.

We will reach to contradiction for both cases. From now on we simply write $\varepsilon$ for $\varepsilon(j)$.

Case 1. In terms of $\left(\mathrm{H}_{x N}\right)$ it is enough to apply the classical method. Combining two inequalities in $(2.10)$, we have

$$
\frac{\alpha}{\left(T-t^{\varepsilon}\right)^{2}} \leqq\left\{H\left(y^{\varepsilon},-p_{y}^{\varepsilon}\right)-H\left(x^{\varepsilon},-p_{y}^{\varepsilon}\right)\right\}+\left\{H\left(x^{\varepsilon},-p_{y}^{\varepsilon}\right)-H\left(x^{\varepsilon}, p_{x}^{\varepsilon}\right)\right\} .
$$

Letting $\varepsilon$ small and applying $\left(\mathrm{H}_{x N}\right)$ and $\left(\mathrm{H}_{p}\right)$, we compute

$$
\begin{aligned}
\frac{\alpha}{\left(T-t^{\varepsilon}\right)^{2}} & \leqq \omega_{2}\left(\left(1+\left|p_{y}^{\varepsilon}\right|\right)\left|x^{\varepsilon}-y^{\varepsilon}\right|\right)+\omega_{1}\left(\left|p_{x}^{\varepsilon}+p_{y}^{\varepsilon}\right|\right) \\
& =\omega_{2}\left(\left|x^{\varepsilon}-y^{\varepsilon}\right|+\frac{1}{\varepsilon^{2}}\left|x^{\varepsilon}-y^{\varepsilon}\right|^{2}\right)+\omega_{1}\left(\left|\beta \nabla f\left(x^{\varepsilon}\right)\right|\right) .
\end{aligned}
$$

Sending $\varepsilon \downarrow 0$ in the above and using $1 / T^{2} \leqq 1 /(T-\hat{t})^{2}$, we obtain $\alpha / T^{2} \leqq \omega_{1}(\beta|\nabla f(\hat{x})|) \leqq \omega_{1}(\beta)$. This is a contradiction for very small $\beta$.

Case 2. By (2.10) we see

$$
\frac{\alpha}{\left(T-t^{\varepsilon}\right)^{2}}+H\left(x^{\varepsilon}, p_{x}^{\varepsilon}\right) \leqq \sigma^{\varepsilon} \leqq H\left(y^{\varepsilon},-p_{y}^{\varepsilon}\right) .
$$

Thus we may assume that $\sigma^{\varepsilon}$ converges to some $-\bar{\tau}$ as $\varepsilon \downarrow 0$ by the local boundedness of $H$. Now, since (2.5), (2.8) and

$$
\lim _{\varepsilon \downarrow 0}\left(p_{x}^{\varepsilon}, \tau^{\varepsilon}, p_{y}^{\varepsilon}, \sigma^{\varepsilon}\right)=\left(\bar{p}+\beta \nabla f(\hat{x}),-\bar{p}, \bar{\tau}+\frac{\alpha}{(T-\hat{t})^{2}},-\bar{\tau}\right)
$$

hold, the definitions of $\bar{D}^{ \pm}$yield

$$
\left\{\begin{array}{l}
\left(\bar{p}+\beta \nabla f(\hat{x}), \bar{\tau}+\frac{\alpha}{(T-\hat{t})^{2}}\right) \in \bar{D}^{+} u^{*}\left(x^{\varepsilon}, t^{\varepsilon}\right), \\
(\bar{p}, \bar{\tau}) \in \bar{D}^{-} v_{*}\left(y^{\varepsilon}, s^{\varepsilon}\right) .
\end{array}\right.
$$

Therefore

$$
\left\{\begin{array}{l}
\bar{\tau}+\frac{\alpha}{(T-\hat{t})^{2}}+H(\hat{x}, \bar{p}+\beta \nabla f(\hat{x})) \leqq 0, \\
\bar{\tau}+H(\hat{x}, \bar{p}) \geqq 0
\end{array}\right.
$$

since $u \in \bar{D}-S U B(H)$ and $v \in \bar{D}-S U P(H)$. Consequently

$$
\frac{\alpha}{T^{2}} \leqq H(\hat{x}, \bar{p})-H(\hat{x}, \bar{p}+\beta \nabla f(\hat{x})) \leqq \omega_{1}(\beta|\nabla f(\hat{x})|) \leqq \omega_{1}(\beta),
$$

which is a contradiction for very small $\beta$. 
Remark 2.7. (1) In general, whenever CP holds, we have $\left\|u_{1}-u_{2}\right\|_{Q} \leqq\left\|\left(\left.u_{1}\right|_{t=0}\right)-\left(\left.u_{2}\right|_{t=0}\right)\right\|_{\mathbf{R}^{n}}$ for any two solutions $u_{1}$ and $u_{2}$ of (1.1), no matter which definition of solutions we use. This is continuous dependence of the solutions on the initial data. Here we write $\|f\|_{U}:=\sup _{U}|f|$ for $f: U \rightarrow \mathbf{R}$.

(2) The term $\beta f(x)$ in the definition of $\Psi$ is added in order that $\mathcal{F}$ attains the maximum in $\left(\mathbf{R}^{n} \times\right.$ $[0, T])^{2}$. If both $u$ and $v$ are periodic in $\mathbf{R}^{n}$, namely $u(x, t)=u\left(x+\sum_{i=1}^{n} e_{i}, t\right)$ and $v(x, t)=v(x+$ $\left.\sum_{i=1}^{n} e_{i}, t\right)$ for some linearly independent $e_{1}, \ldots, e_{n} \in \mathbf{R}^{n}$, the function $\mathcal{F}$ attains the maximum without $\beta f(x)$, and then we have $p_{x}^{\varepsilon}=-p_{y}^{\varepsilon}$. Therefore it is unnecessary to assume $\left(\mathrm{H}_{p}\right)$ in this periodic case.

Corollary 2.8 (uniqueness of $\bar{D}$-solutions). Assume that $H$ satisfies $\left(\mathrm{H}_{p}\right)$ and $\left(\mathrm{H}_{x N}\right)$. Then there exists at most one $\bar{D}$-solution of (HJ) and it is continuous.

Proof. Let $u, v \in \bar{D}-S O L\left(H, u_{0}\right)$. Applying Theorem 2.6 to a subsolution $u$ and a supersolution $v$, we get $u^{*} \leqq v_{*}$ in $Q$. Next changing roles of $u$ and $v$, we also see $v^{*} \leqq u_{*}$ in $Q$. Hence it follows that $u^{*} \leqq v_{*} \leqq v^{*} \leqq u_{*}$ in $Q$, which yields our claim.

The assumption $\left(\mathrm{H}_{x N}\right)$ was used only in Case1 in the proof of Theorem 2.6 for the situation that elements in $D^{+} u^{*}$ and $D^{-} v_{*}$ are unbounded. For any Lipschitz continuous function $w$ in $Q$, we have $|p| \leqq \operatorname{Lip}[w]$ and $\tau \leqq \operatorname{Lip}[w]$ whenever $(p, \tau) \in D^{+} w(\hat{x}, \hat{t})$ or $(p, \tau) \in D^{-} w(\hat{x}, \hat{t})$, where $\operatorname{Lip}[w]$ stands for the Lipschitz constant of $w$. Therefore it is unnecessary to assume $\left(\mathrm{H}_{x N}\right)$ in order to prove CP when one of solutions is Lipschitz continuous.

Theorem 2.9 (CP-Lipschitz version). Assume that $H$ satisfies $\left(\mathrm{H}_{p}\right)$. Let $u$ and $v: Q_{0} \rightarrow \mathbf{R}$ be, respectively, bounded from above and bounded from below in $Q_{0}$. Assume that $u \in \bar{D}$-SUB $(H)$ and $v \in \bar{D}$-SUP $(H)$. Furthermore assume that either $u$ or $v$ is (space-time) Lipschitz continuous in $Q$. If $u^{*}(\cdot, 0) \leqq v_{*}(\cdot, 0)$ in $\mathbf{R}^{n}$, then $u^{*} \leqq v_{*}$ in $Q$.

As mentioned in Remark $2.7(2)$, the assumption $\left(\mathrm{H}_{p}\right)$ is unnecessary for the periodic case. It is not difficult to find that this version of $\mathrm{CP}$ applies to (1.3).

\section{Existence results}

\subsection{Unique existence of envelope solutions}

We adopted $\bar{D}$-solutions as a proper definition in Section 2.1 in order to guarantee the uniqueness of solutions of (HJ) with (1.3) and $u_{0} \equiv 0$, but the existence turns out to be an issue for a discontinuous Hamiltonian. We give two examples to show the non-existence of $\bar{D}$-solutions.

Example 3.1. Let us consider (HJ) with (1.15) and $u_{0} \equiv 0$. Then $u \equiv 0$ is a subsolution but is not a $\bar{D}$-supersolution. Also, one observes that $u^{\varepsilon}(x, t)=c t I^{\varepsilon}(x)$ with (1.7) is a $\bar{D}$-supersolution but is not a subsolution for each $\varepsilon>0$. Therefore, if there would exist a $\bar{D}$-solution $v$, then $0 \leqq v_{*} \leqq v^{*} \leqq u^{\varepsilon}$ in $Q$ by Theorem 2.9. Sending $\varepsilon \downarrow 0$, we see $0 \leqq v_{*} \leqq v^{*} \leqq c t I(x)$. Hence $v_{*} \equiv 0$ in $Q$, which contradicts the fact that 0 is not a $\bar{D}$-supersolution. (See Figure 3.)

Example 3.2. Let us consider (HJ) with (1.3) and $u_{0} \equiv 0$. The intuitive solution $u(x, t)=c(t-|x|)_{+}$ is a subsolution but is not a $\bar{D}$-supersolution because $(p, c) \in \bar{D}^{-} u(0,1)(|p|=c)$ and $c-|p|<c I(0)$. Now we think of approximate problems

$$
(\varepsilon . \mathrm{HJ}) \begin{cases}\partial_{t} u+H^{\varepsilon}(x, \nabla u)=0 & \text { in } Q, \\ \left.u\right|_{t=0}=u_{0} & \text { in } \mathbf{R}^{n},\end{cases}
$$

where $H^{\varepsilon}$ is given by (1.6). Since we can write $H^{\varepsilon}(x, p)=-\max _{a \in \bar{B}_{1}(0)}\langle a, p\rangle-c I^{\varepsilon}(x)$, the representation formula by the optimal control theory (see Section 5.1 for more details) implies that $u^{\varepsilon}$ given by

$$
u^{\varepsilon}(x, t)=\sup _{\alpha \in \mathcal{A}} \int_{0}^{t} c I^{\varepsilon}\left(X^{\alpha}(s)\right) d s
$$



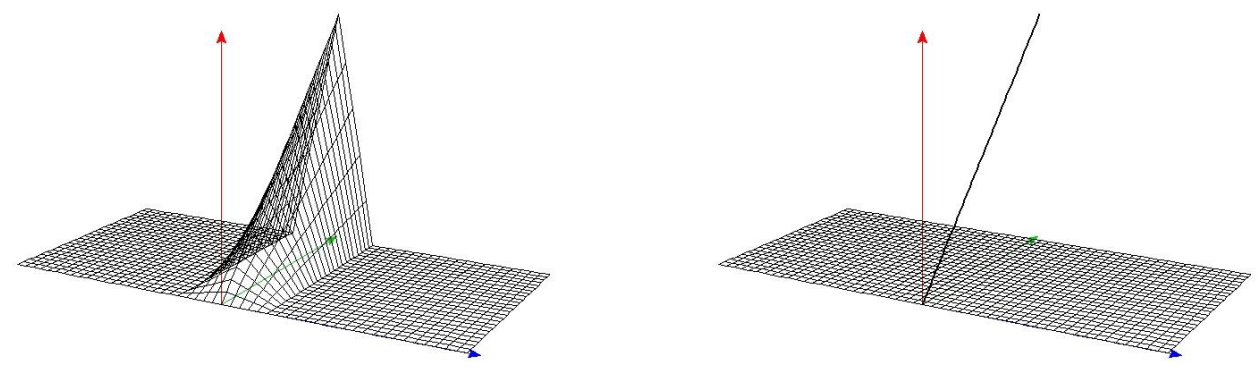

Figure 3: $\bar{D}$-supersolutions $t I^{\varepsilon}(x)$ of $\partial_{t} u=I(x), u_{0} \equiv 0$ (the left) and their limit $t I(x)$ (the right). The latter is an envelope solution.

is a unique viscosity solution of $\left(\varepsilon\right.$.HJ). Here $\mathcal{A}=\left\{\alpha:[0, T] \rightarrow \bar{B}_{1}(0)\right.$, measurable $\}$ and $X^{\alpha}(s)$ is the solution of the state equation: $X^{\prime}(s)=\alpha(s)$ in $(0, t), X(0)=x$. In other words, $X^{\alpha}(s)$ describes a trajectory which leaves at time 0 from $x$ and moves at velocity 1 or less. In this case for each $x \in \mathbf{R}^{n}$ the optimal control is the one that leads to a straight trajectory before it comes to the origin and stays there after that moment. A direct calculation yields

$$
\begin{aligned}
u^{\varepsilon}(x, t) / c= \begin{cases}\left(1-\frac{|x|}{\varepsilon}\right) t+\frac{t^{2}}{2 \varepsilon} & (t \leqq|x|), \\
t-\frac{|x|^{2}}{2 \varepsilon} & (t \geqq|x|),\end{cases} \\
\text { and } u^{\varepsilon}(x, t) / c= \begin{cases}0 & \text { for }|x| \leqq \varepsilon, \\
\frac{(t-|x|+\varepsilon)^{2}}{2 \varepsilon} & (t \leqq|x|-\varepsilon), \\
t-|x|+\frac{\varepsilon}{2} & (t \geqq|x|),\end{cases}
\end{aligned}
$$

The inequality $H \geqq H^{\varepsilon}$ implies that each $u^{\varepsilon}$ is a $\bar{D}$-supersolution of the original (HJ). However, since $u^{\varepsilon} \downarrow u$ as $\varepsilon \downarrow 0$, it is shown by the similar argument in the previous example that there is no $\bar{D}$-solution of (HJ). (See Figure 4.)
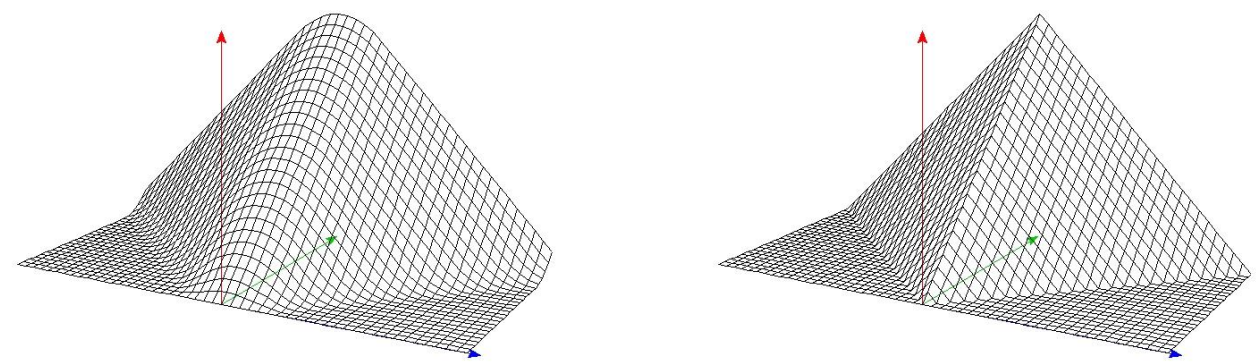

Figure 4: $\bar{D}$-supersolutions $u^{\varepsilon}$ of $\partial_{t} u-|\nabla u|=I(x), u_{0} \equiv 0$ (the left) and their limit $(t-|x|)_{+}($the right). The latter is an envelope solution.

For (HJ) with (1.3) and $u_{0} \equiv 0$, there are infinite $D$-solutions while there is no $\bar{D}$-solution. This suggests that we must define another proper notion of solutions. 
Definition 3.3 (envelope solutions). Let $\mathcal{S}$ be a nonempty subset of $\bar{D}-S U P(H)$. If $v:=\inf _{w \in \mathcal{S}} w$ is bounded from below in $Q$, it is said to be an envelope viscosity supersolution of (1.1). Let e.SUP $(H)$ denote the set of all such solutions. If $v$ is also an envelope viscosity subsolution (write $v \in$ e.SUB $(H)$ ), i.e., $v=\sup _{w \in \mathcal{T}} w$ for some $\mathcal{T} \subset \bar{D}$-SUB $(H)$ and $v$ is bounded from above in $Q$, we call it an envelope viscosity solution. Set e.SOL $(H):=$ e.SUB $(H) \cap$ e.SUP $(H)$. We also define e.SUB $\left(H, u_{0}\right)$, e.SUP $\left(H, u_{0}\right)$ and e.SOL $\left(H, u_{0}\right)$ as the sets of all (sub, super)solutions of (HJ) similarly as before.

Remark 3.4. (1) The function $\operatorname{ct} I(x)$ is an envelope solution in Example 3.1 and $c(t-|x|)_{+}$is an envelope solution in Example 3.2. Also, Example 3.1 suggests that our envelope solution is not always continuous.

(2) Since standard viscosity supersolutions have stability, that is, the infimum of them is still a supersolution (see for instance [9, Lemma 4.2]), the class of solutions does not become large by taking infimum. As for $\bar{D}$-supersolutions, however, we observed that $\inf _{\varepsilon>0} u^{\varepsilon} \notin \bar{D}$-SUP(H) in Examples 3.1 and 3.2. In other words, stability under infimum does not hold for $\bar{D}$-supersolutions in general. By contrast, our envelope supersolutions have such stability by the definition. We also learn by Example 3.2 that $\bar{D}$-supersolutions are not stable even under the uniform limit.

(3) We have $\bar{D}$-SUP $(H) \subset \operatorname{e.SUP}(H) \subset S U P(H)$, but $e . S U P(H) \subset D$-SUP $(H)$ does not hold in general. (See Figure 5.) The function $\operatorname{ct} I(x)$ in Example 3.1 is its counter-example. If $H$ is lower semicontinuous, then e.SUB $(H)=S U B(H)$.

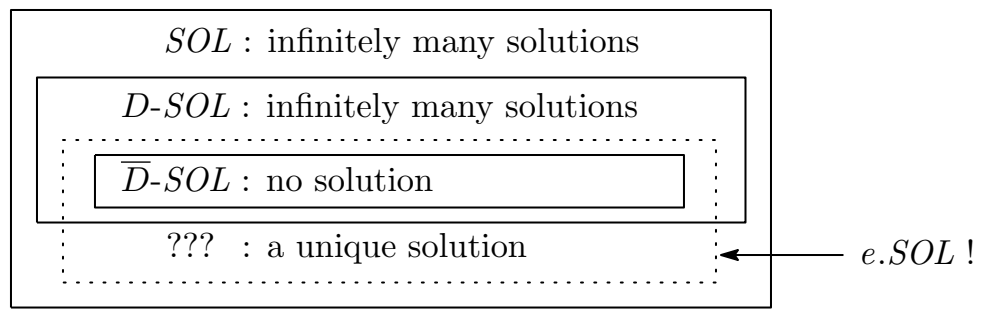

Figure 5: The notion of envelope solutions.

As was pointed out in Remark 3.4 (2) we do not have the stability under infimum for $\bar{D}$-supersolutions in general, but it is shown that the infimum of finitely many $\bar{D}$-supersolutions is still a $\bar{D}$-supersolution.

Proposition 3.5 (stability under infimum of finitely many solutions). Let $u_{i} \in \bar{D}-S U P(H)$ for all $i \in\{1,2, \ldots, M\}$. Then $u:=\min _{i=1}^{M} u_{i} \in \bar{D}-S U P(H)$.

Proof. We first remark that $u_{*}=\min _{i=1}^{M}\left(u_{i}\right)_{*}$. Fix $(\hat{x}, \hat{t}) \in Q,(p, \tau) \in \bar{D}^{-} u_{*}(\hat{x}, \hat{t})$ and take a defining sequence $\left(x_{m}, t_{m}\right) \in Q,\left(p_{m}, \tau_{m}\right) \in D^{-} u_{*}\left(x_{m}, t_{m}\right)(m \in \mathbf{N})$. Then we have $\lim _{m \rightarrow \infty} u_{*}\left(x_{m}, t_{m}\right)=$ $u_{*}(\hat{x}, \hat{t})$, and there exists a subsequence $\{m(k)\}_{k \in \mathbf{N}}$ of $\{m\}_{m \in \mathbf{N}}$ such that

$$
u_{*}\left(x_{m(k)}, t_{m(k)}\right)=\left(u_{i}\right)_{*}\left(x_{m(k)}, t_{m(k)}\right) \quad(\forall k \in \mathbf{N})
$$

for some $i \in\{1,2, \ldots, M\}$. Observe that

$$
\begin{aligned}
u_{*}(\hat{x}, \hat{t}) & =\lim _{m \rightarrow \infty} u_{*}\left(x_{m}, t_{m}\right) \\
& =\lim _{k \rightarrow \infty} u_{*}\left(x_{m(k)}, t_{m(k)}\right) \\
& =\lim _{k \rightarrow \infty}\left(u_{i}\right)_{*}\left(x_{m(k)}, t_{m(k)}\right) \geqq\left(u_{i}\right)_{*}(\hat{x}, \hat{t}) .
\end{aligned}
$$

Therefore, it follows that $u_{*}(\hat{x}, \hat{t})=\left(u_{i}\right)_{*}(\hat{x}, \hat{t})$. We thus have

$$
\left(p_{m(k)}, \tau_{m(k)}\right) \in D^{-}\left(u_{i}\right)_{*}\left(x_{m(k)}, t_{m(k)}\right), \quad \lim _{k \rightarrow \infty}\left(u_{i}\right)_{*}\left(x_{m(k)}, t_{m(k)}\right)=\left(u_{i}\right)_{*}(\hat{x}, \hat{t}),
$$

and hence $(p, \tau) \in \bar{D}^{-}\left(u_{i}\right)_{*}(\hat{x}, \hat{t})$. Since $u_{i} \in \bar{D}-S U P(H)$, we deduce that $\tau+H(\hat{x}, p) \geqq 0$. 
We now present the uniqueness result for envelope solutions.

Proposition 3.6 (uniqueness of envelope solutions). Assume that $H$ satisfies $\left(\mathrm{H}_{p}\right)$ and $\left(\mathrm{H}_{x N}\right)$. Then there exists at most one envelope solution of (HJ). Moreover if $H$ is lower semicontinuous, the unique envelope solution is upper semicontinuous.

Proof. Let $u, v \in e . S O L\left(H, u_{0}\right)$. We first use the fact that $u \in e . S U B\left(H, u_{0}\right)$ and $v \in e . S U P\left(H, u_{0}\right)$. By the definition of envelope sub- and supersolutions there exists some $\mathcal{T} \subset \bar{D}-S U B\left(H, u_{0}\right)$ and $\mathcal{S} \subset$ $\bar{D}-S U P\left(H, u_{0}\right)$ such that $u=\sup _{w \in \mathcal{T}} w$ and $v=\inf _{W \in \mathcal{S}} W$. Then applying Theorem 2.6 to $w \in \mathcal{T}$ and $W \in \mathcal{S}$, we get $w^{*} \leqq W_{*}$ in $Q$, which yields $u \leqq v$ in $Q$. Next changing roles of $u$ and $v$, we also see $v \leqq u$ in $Q$, and hence our first claim is proved.

If $H$ is lower semicontinuous, we apply Theorem 2.6 to $u$ and $W \in \mathcal{S}$. Then we deduce that $u^{*} \leqq$ $W_{*}$ in $Q$, hence that $u^{*} \leqq v$ in $Q$. Since we also have $v^{*} \leqq u$ in $Q$, it follows that $u^{*} \leqq v \leqq v^{*} \leqq u$ in $Q$, and so our second claim follows.

We next consider the existence of envelope solutions. We will construct the solution as the infimum of $u^{\varepsilon}$, which are solutions for "good" Hamiltonians $H^{\varepsilon}$ approximating $H$. Here "good" means that comparison and existence properties are ensured for solutions. We use the following assumption.

$\left(\mathrm{H}_{\varepsilon}\right)$ There exists a family $\left\{H^{\varepsilon}\right\}_{\varepsilon>0} \subset C\left(\mathbf{R}^{n} \times \mathbf{R}^{n}\right)$ such that $H^{\varepsilon} \uparrow H(\varepsilon \downarrow 0)$ pointwise, and for all $\varepsilon>0$ and $u_{0} \in B U C\left(\mathbf{R}^{n}\right)$ the following two statements hold.

(i) If $w^{\varepsilon} \in S U B\left(H^{\varepsilon}, u_{0}\right)$ and $v^{\varepsilon} \in S U P\left(H^{\varepsilon}, u_{0}\right)$, then $\left(w^{\varepsilon}\right)^{*} \leqq\left(v^{\varepsilon}\right)_{*}$ in $Q$.

(ii) There exists a bounded solution $u^{\varepsilon} \in S O L\left(H^{\varepsilon}, u_{0}\right)$.

If there is some $u^{\varepsilon} \in S O L\left(H^{\varepsilon}, u_{0}\right)$, it is automatically continuous and a unique solution by the comparison (i). Also, $H$ satisfying $\left(\mathrm{H}_{\varepsilon}\right)$ is lower semicontinuous.

We here recall the Perron's method for constructing standard viscosity solutions. (See for instance [9, Theorem 4.1.].) Let $v \in S U B\left(H, u_{0}\right), V \in S U P\left(H, u_{0}\right)$ and $v \leqq V$ in $Q$. Then $u$ defined by

$$
u:=\sup \left\{w \in S U B\left(H, u_{0}\right) \mid v \leqq w \leqq V \text { in } Q\right\}
$$

is a viscosity solution of (HJ). Functions $v$ and $V$ are called respectively a lower barrier and an upper barrier. One can construct these barriers for all $u_{0} \in B U C\left(\mathbf{R}^{n}\right)$ provided that $H(x, p)$ is bounded locally in $p$ (see [15, Lemma 4.3.4.]), i.e.,

$\left(\mathrm{H}_{m}\right) m(\rho):=\sup \left\{|H(x, p)| \mid(x, p) \in \mathbf{R}^{n} \times \bar{B}_{\rho}(0)\right\}<\infty$ for all $\rho \geqq 0$.

Proposition 3.7 (existence). Assume that $H$ satisfies $\left(\mathrm{H}_{\varepsilon}\right)$ and $\left(\mathrm{H}_{m}\right)$. Let $u^{\varepsilon} \in S O L\left(H^{\varepsilon}, u_{0}\right)$ in $\left(\mathrm{H}_{\varepsilon}\right)$. Then $\bar{u}:=\inf _{\varepsilon>0} u^{\varepsilon}$ is an envelope solution of $(\mathrm{HJ})$.

We call $\bar{u}$ constructed in this way a solution approximated from above. By the definition $\bar{u}$ is upper semicontinuous.

Proof. We first show that $u^{\varepsilon}$ is monotone in $\varepsilon$. Let $0<\varepsilon<\varepsilon^{\prime}$. Then $u^{\varepsilon} \in S U B\left(H^{\varepsilon}, u_{0}\right)$, and also we see $u^{\varepsilon^{\prime}} \in S U P\left(H^{\varepsilon^{\prime}}, u_{0}\right) \subset S U P\left(H^{\varepsilon}, u_{0}\right)$ since $H^{\varepsilon^{\prime}} \leqq H^{\varepsilon}$. Therefore we conclude that $u^{\varepsilon} \leqq u^{\varepsilon^{\prime}}$ by the comparison. This monotonicity implies that $\bar{u}=\lim \sup _{\varepsilon \downarrow 0}^{*} u^{\varepsilon}$ and that $\bar{u}$ is bounded from above. Now, we are able to take an upper semicontinuous lower barrier $v \in S U B\left(H, u_{0}\right)$ on account of the assumption $\left(\mathrm{H}_{m}\right)$. Since $v \in S U B\left(H^{\varepsilon}, u_{0}\right)$, we see by the comparison that $v \leqq u^{\varepsilon}$, and so $v \leqq \bar{u}$. We also find that $\bar{u}$ is bounded from below.

Since $u^{\varepsilon} \in S U B\left(H^{\varepsilon}\right)$, we see $\bar{u} \in S U B\left(\liminf _{* \varepsilon \downarrow 0} H^{\varepsilon}\right)=S U B(H)$ by the stability of viscosity subsolutions. Also, $\bar{u}$ is an envelope supersolution of (HJ) because $\bar{u}=\inf _{\varepsilon>0} u^{\varepsilon}$ and $u^{\varepsilon} \in S U P\left(H^{\varepsilon}\right) \subset$ $\bar{D}-S U P(H)$. We finally show that $\bar{u}$ is continuous at the initial time. Take any $x \in \mathbf{R}^{n}$ and $(y, s) \in Q_{0}$. Then $v(y, s)-u_{0}(x) \leqq \bar{u}(y, s)-u_{0}(x) \leqq u^{\varepsilon}(y, s)-u_{0}(x)$ and both $v(y, s)$ and $u^{\varepsilon}(y, s)$ converge to $u_{0}(x)$ as $(y, s) \rightarrow(x, 0)$. As a result we deduce that $\bar{u}(y, s) \rightarrow u_{0}(x)$. 
Remark 3.8. For any subset $L \subset \mathbf{R}^{N}$ and $h^{\varepsilon}: L \rightarrow \mathbf{R}(\varepsilon>0)$ we denote the upper relaxed limit (resp. lower relaxed limit) by $\bar{h}=\limsup _{\varepsilon \downarrow 0}^{*} h^{\varepsilon}\left(\operatorname{resp} . \underline{h}=\liminf _{* \varepsilon \downarrow 0} h^{\varepsilon}\right): \bar{L} \rightarrow \mathbf{R} \cup\{ \pm \infty\}$, which is as follows:

$$
\begin{aligned}
\bar{h}(z) & :=\limsup _{(\varepsilon, y) \rightarrow(0, z)} h^{\varepsilon}(y)=\lim _{\delta \downarrow 0} \sup \left\{h^{\varepsilon}(y) \mid y \in \bar{B}_{\delta}(z) \cap L, 0<\varepsilon<\delta\right\} \\
\text { (resp. } \underline{h}(z) & \left.:=\liminf _{(\varepsilon, y) \rightarrow(0, z)} h^{\varepsilon}(y)=\liminf _{\delta \downarrow 0}\left\{h^{\varepsilon}(y) \mid y \in \bar{B}_{\delta}(z) \cap L, 0<\varepsilon<\delta\right\}\right) \quad(z \in \bar{L}) .
\end{aligned}
$$

The following properties are easily seen by the definition: If $h^{\varepsilon} \equiv h$, then $\bar{h}=h^{*}$ and $\underline{h}=h_{*}$. If $h^{\varepsilon} \downarrow h\left(\right.$ resp. $\left.h^{\varepsilon} \uparrow h\right)$ monotonously, then $\bar{h}=h^{*}\left(\operatorname{resp} . \underline{h}=h_{*}\right)$. Also, $\bar{h}=\limsup _{\varepsilon \downarrow 0}^{*}\left(h^{\varepsilon}\right)^{*}$ and $\underline{h}=\liminf \operatorname{in\downarrow }_{* 0}\left(h^{\varepsilon}\right)_{*}$ in general.

We next present examples of $H$ which satisfies the assumption $\left(\mathrm{H}_{\varepsilon}\right)$. In order to obtain the comparison and existence properties in $\left(\mathrm{H}_{\varepsilon}\right)$, it is sufficient that each $H^{\varepsilon}$ satisfies $\left(\mathrm{H}_{p}\right),\left(\mathrm{H}_{x}\right)$ and $\left(\mathrm{H}_{m}\right)$.

Example 3.9. If $H$ is lower semicontinuous and bounded in $\mathbf{R}^{n} \times \mathbf{R}^{n}$, then $\left(\mathrm{H}_{\varepsilon}\right)$ is fulfilled. In this case we take $H^{\varepsilon}$ as the inf-convolution of $H$ over $\mathbf{R}^{n} \times \mathbf{R}^{n}$. (See below about sup- and inf-convolution.) Each $H^{\varepsilon}$ satisfies $\left(\mathrm{H}_{p}\right)$ and $\left(\mathrm{H}_{x}\right)$ since it is globally Lipschitz continuous, and $\left(\mathrm{H}_{m}\right)$ is clear from the boundedness of $H^{\varepsilon}$.

Example 3.10. Let $H$ have the form of (1.9) with $r \in B U S C\left(\mathbf{R}^{n}\right)$. Assume that $H_{0}$ is uniformly continuous in $\mathbf{R}^{n} \times \mathbf{R}^{n}$ and satisfies $\left(\mathrm{H}_{m}\right)$. Then $\left(\mathrm{H}_{\varepsilon}\right)$ is fulfilled. The conditions $\left(\mathrm{H}_{p}\right),\left(\mathrm{H}_{x}\right),\left(\mathrm{H}_{m}\right)$ are all satisfied by $H^{\varepsilon}(x, p)=H_{0}(x, p)-r^{\varepsilon}(x)$, where $r^{\varepsilon}$ is the sup-convolution of $r$.

Remark 3.11 (sup- and inf-convolution). For bounded $f: \mathbf{R}^{N} \rightarrow \mathbf{R}$ and $\varepsilon>0$ we define the supconvolution $f^{\varepsilon}$ (resp. inf-convolution $f_{\varepsilon}$ ) of $f$ by

$$
f^{\varepsilon}(x):=\sup _{y \in \mathbf{R}^{N}}\left\{f(y)-\frac{1}{2 \varepsilon}|x-y|^{2}\right\} . \quad\left(\operatorname{resp} . f_{\varepsilon}(x):=\inf _{y \in \mathbf{R}^{N}}\left\{f(y)+\frac{1}{2 \varepsilon}|x-y|^{2}\right\} .\right)
$$

The following properties are easily found, and so we omit the verification.

- $-\|f\|_{\mathbf{R}^{n}} \leqq f \leqq f^{\delta} \leqq f^{\varepsilon} \leqq\|f\|_{\mathbf{R}^{n}}$ for $0<\delta<\varepsilon$.

- $f^{\varepsilon}$ is Lipschitz continuous in $\mathbf{R}^{N}$.

- If $f$ is upper semicontinuous, then $f^{\varepsilon}(x) \downarrow f(x)(\varepsilon \downarrow 0)$ for each $x \in \mathbf{R}^{N}$.

- If $f$ is uniformly continuous, then $f^{\varepsilon}$ converges to $f$ uniformly in $\mathbf{R}^{N}$.

We mainly use these convolutions in order to approximate semicontinuous functions by Lipschitz continuous ones.

Combining Proposition 3.6 and Proposition 3.7, we obtain the unique existence result.

Theorem 3.12 (unique existence-general version). Assume that $H$ satisfies $\left(\mathrm{H}_{p}\right),\left(\mathrm{H}_{x N}\right),\left(\mathrm{H}_{\varepsilon}\right)$ and $\left(\mathrm{H}_{m}\right)$. Then $\bar{u}$, a solution approximated from above, is a unique envelope solution of (HJ).

If we do not accept the assumption $\left(\mathrm{H}_{x N}\right)$, Lipschitz continuities of solutions are needed for CP.

Theorem 3.13 (unique existence-Lipschitz version). Assume that $H$ satisfies $\left(\mathrm{H}_{p}\right),\left(\mathrm{H}_{\varepsilon}\right)$ and $\left(\mathrm{H}_{m}\right)$. Let $u^{\varepsilon} \in S O L\left(H^{\varepsilon}, u_{0}\right)$ in $\left(\mathrm{H}_{\varepsilon}\right)$ and assume that $u^{\varepsilon}(\varepsilon>0)$ and $\inf _{\varepsilon>0} u^{\varepsilon}$ are Lipschitz continuous in $Q$. Then $\bar{u}$, a solution approximated from above, is a unique envelope solution of (HJ).

Proof. We only need to show the uniqueness. Let $v \in e . S O L\left(H, u_{0}\right)$. An analogue of the proof of Proposition 3.6 works and yields the inequality $\bar{u} \leqq v_{*}$ in $Q$ (but we use Theorem 2.9 here). Next, since $v \in S U B\left(H, u_{0}\right), u^{\varepsilon} \in \bar{D}-S U P\left(H, u_{0}\right)$ and $u^{\varepsilon}$ is Lipschitz continuous, Theorem 2.9 yields that $v^{*} \leqq u^{\varepsilon}$ in $Q$, and so $v^{*} \leqq \bar{u}$ in $Q$. Thus $\bar{u}=v$. 
Remark 3.14. Let $u \in e . S O L\left(H, u_{0}\right)$ and $\left(\mathrm{H}_{p}\right)$ hold. If there exists some $\mathcal{T} \subset \bar{D}$-SUB $\left(H, u_{0}\right) \cap B L i p(Q)$ (resp. $\left.\mathcal{S} \subset \bar{D}-S U P\left(H, u_{0}\right) \cap B L i p(Q)\right)$ such that $u=\sup _{v \in \mathcal{T}} v\left(\operatorname{resp} . \quad u=\inf _{w \in \mathcal{S}} w\right)$, then $u$ is the minimal (resp. maximal) envelope solution. These facts are easily shown by using Theorem 2.9.

Remark 3.15. If the Lipschitz constants of $u^{\varepsilon}$ are estimated uniformly in $\varepsilon$, then $\bar{u}=\inf _{\varepsilon>0} u^{\varepsilon}$ is also Lipschitz continuous (provided that $u^{\varepsilon}$ are bounded uniformly in $\varepsilon$ ). In general, if $u^{\varepsilon}$ have their modulus $\omega \in \mathcal{M}$ independent of $\varepsilon$, their infimum $\bar{u}$ also has the same $\omega$ as its modulus.

Example 3.16. In Example 3.2 the function $u(x, t)=c(t-|x|)_{+}$is a unique envelope solution by Theorem 3.13 since $u^{\varepsilon}$ and $u$ are Lipschitz continuous. In Example 3.1, on the other hand, the envelope solutions are not unique in that $v^{\alpha}(x, t)=\alpha t I(x)(\alpha \in(0, c])$ are all envelope solutions. Let us show this claim. It is easily seen that they are all subsolutions. Set $v^{\alpha, \varepsilon}(x, t):=\alpha t\left\{(1-\sqrt{|x| / \varepsilon})_{+}\right\}^{2}$ for $\varepsilon>0$. (See Figure 6.) Then one observes that $v^{\alpha, \varepsilon} \in \bar{D}-S U P(H, 0)$ since $\partial_{t} v^{\alpha, \varepsilon} \geqq 0$ and $\bar{D}^{-} v^{\alpha, \varepsilon}(0, \hat{t})=\emptyset$. Hence the equality $v^{\alpha}=\inf _{\varepsilon>0} v^{\alpha, \varepsilon}$ implies our claim. Moreover $v^{0} \equiv 0$ is also an envelope solution since $v^{0}=\inf _{\alpha \in(0, c], \varepsilon>0} v^{\alpha, \varepsilon}$. The function $v^{c}$ is the maximal envelope solution by Remark 3.14 because $v^{c}(x, t)=\inf _{\varepsilon>0} c t I^{\varepsilon}(x)$ and $c t I^{\varepsilon}(x) \in \bar{D}-S U P(H, 0) \cap B \operatorname{Bip}(Q)$, where $I^{\varepsilon}(x):=(1-|x| / \varepsilon)_{+}$. Also, $v^{0} \equiv 0$ is the minimal envelope solution.

For a general initial data $u_{0} \in B U C\left(\mathbf{R}^{n}\right)$, it is also seen that $u_{0}(x)+\alpha t I(x)(\alpha \in[0, c])$ are all envelope solutions. Uniqueness, therefore, always goes wrong for the initial-value problem with the equation $\partial_{t} u=c I(x)$. Such bad behavior can happen when a Hamiltonian is non-coercive. Indeed, we establish the uniqueness result for coercive Hamiltonians in the next subsection (Theorem 3.22).

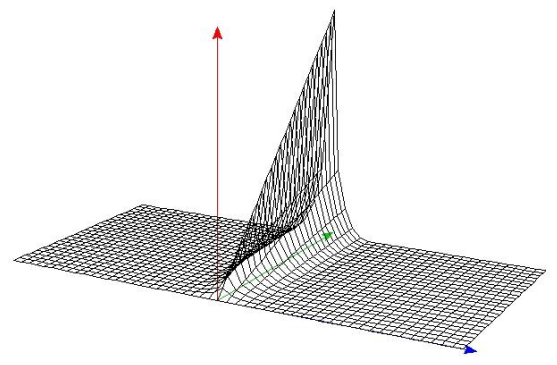

Figure 6: $\bar{D}$-supersolutions $v^{\alpha, \varepsilon}$ of $\partial_{t} u=I(x), u_{0} \equiv 0$.

\subsection{Coercive Hamiltonians}

In order to apply Theorem 3.13, we need to know what conditions guarantee the Lipschitz continuities of $u^{\varepsilon}$ and $\inf _{\varepsilon>0} u^{\varepsilon}$. We therefore consider in this subsection whether the solutions preserve the continuity of initial data. For continuous Hamiltonians it is known that such preserving properties hold if they are coercive, namely

$$
\lim _{|p| \rightarrow \infty} \inf _{x \in \mathbf{R}^{n}} H(x, p)=\infty \quad \text { or } \quad \lim _{|p| \rightarrow \infty} \sup _{x \in \mathbf{R}^{n}} H(x, p)=-\infty .
$$

The coercivity of $H$ is equivalent to $\left(\mathrm{H}_{R+}\right)$ or $\left(\mathrm{H}_{R-}\right)$ below.

$\left(\mathrm{H}_{R+}\right) R_{+}(m):=\sup \left\{|p| \mid \exists x \in \mathbf{R}^{n}, H(x, p) \leqq m\right\}<\infty$ for all $m \geqq 0$.

$\left(\mathrm{H}_{R-}\right) R_{-}(m):=\sup \left\{|p| \mid \exists x \in \mathbf{R}^{n}, H(x, p) \geqq-m\right\}<\infty$ for all $m \geqq 0$.

Here we use the convention that $\sup \emptyset=0$. We first present Lipschitz continuity and BUC (bounded uniform continuity) preserving properties for continuous Hamiltonians. These results are more or less known. See for example [7], where they discussed for a.e. (sub)solutions. We give here a proof based 
on the theory of viscosity solutions without using a.e. solutions. By using these results we establish our preserving properties for discontinuous Hamiltonians via approximation by continuous ones.

For a function $u: Q \rightarrow \mathbf{R}$, we define

$$
\begin{aligned}
& \operatorname{Lip}_{t}[u]:=\sup _{x \in \mathbf{R}^{n}} \sup _{\substack{t, s \in(0, T) \\
t \neq s}} \frac{|u(x, t)-u(x, s)|}{|t-s|}, \\
& \operatorname{Lip}_{x}[u]:=\sup _{t \in(0, T)} \sup _{\substack{x, y \in \mathbf{R}^{n} \\
x \neq y}} \frac{|u(x, t)-u(y, t)|}{|x-y|} .
\end{aligned}
$$

Proposition 3.17 (Lipschitz continuity preserving property). Assume that $H$ satisfies $\left(\mathrm{H}_{p}\right)$, $\left(\mathrm{H}_{x}\right)$, $\left(\mathrm{H}_{m}\right)$ and $\left(\mathrm{H}_{R+}\right)$. Let $u_{0} \in \operatorname{BLip}\left(\mathbf{R}^{n}\right)$ and $u \in S O L\left(H, u_{0}\right)$. Then $u \in B L i p(Q)$ with the Lipschitz constant satisfying

$$
\operatorname{Lip}_{t}[u] \leqq m, \quad \operatorname{Lip}_{x}[u] \leqq R_{+}(m),
$$

where $m:=m\left(\operatorname{Lip}\left[u_{0}\right]\right)$ and $m(\cdot)$ is the function in $\left(\mathrm{H}_{m}\right)$.

The assumption $\left(\mathrm{H}_{R+}\right)$ is able to be replaced by $\left(\mathrm{H}_{R-}\right)$. (The same is valid for Proposition 3.20.)

Proof. We first remark that $w(x, t):=u_{0}(x)+m t \in S U P\left(H, u_{0}\right)$. Take any $(\hat{x}, \hat{t}) \in Q, h \in(0, T-\hat{t})$ and define

$$
\tilde{u}(x, t):= \begin{cases}w(x, t) & (t \in[0, h]), \\ u(x, t-h)+m h & (t \in(h, T)) .\end{cases}
$$

We claim $\tilde{u} \in S U P\left(H, u_{0}\right)$. Let $(p, \tau) \in D^{-} \tilde{u}(\hat{x}, \hat{t})$. Then it follows easily that $\tau+H(\hat{x}, p) \geqq 0$ when $\hat{t} \neq h$, and so we only consider the case $\hat{t}=h$. Since $u \in S U B\left(H, u_{0}\right)$ and $w \in S U P\left(H, u_{0}\right)$, we see by the comparison principle that $u \leqq w$ in $Q$. Take $(x, t) \in \mathbf{R}^{n} \times(h, T)$, and substitute $(x, t-h)$ into the inequality. Then we find $u(x, t-h) \leqq u_{0}(x)+m(t-h)$, namely $\tilde{u}(x, t) \leqq w(x, t)$. This implies the relation $D^{-} \tilde{u}(\hat{x}, h) \subset D^{-} w(\hat{x}, h)$, and hence our claim follows from $w \in S U P\left(H, u_{0}\right)$.

Applying the comparison principle to $u \in S U B\left(H, u_{0}\right)$ and $\tilde{u} \in S U P\left(H, u_{0}\right)$, we obtain $u \leqq \tilde{u}$ in $Q$. In particular, we have $u(\hat{x}, \hat{t}+h) \leqq \tilde{u}(\hat{x}, \hat{t}+h)=u(\hat{x}, \hat{t})+m h$, that is

$$
\frac{u(\hat{x}, \hat{t}+h)-u(\hat{x}, \hat{t})}{h} \leqq m
$$

By the similar argument we also deduce

$$
\frac{u(\hat{x}, \hat{t}+h)-u(\hat{x}, \hat{t})}{h} \geqq-m
$$

from the fact that $u_{0}(x)-m t \in S U B\left(H, u_{0}\right)$ and so on. Thus $\operatorname{Lip}_{t}[u] \leqq m$ is proved.

We next estimate $\operatorname{Lip}_{x}[u]$. Take any $(p, \tau) \in D^{+} u(\hat{x}, \hat{t})$. Since the estimate $\operatorname{Lip}_{t}[u] \leqq m$ implies $|\tau| \leqq m$, we see from $u \in S U B(H)$ that $H(\hat{x}, p) \leqq-\tau \leqq m$, hence that $|p| \leqq R_{+}(m)$ by $\left(\mathrm{H}_{R+}\right)$. This observation means

$$
\sup _{\substack{(\hat{x}, \hat{t}) \in Q \\(p, \tau) \in D^{+} u(\hat{x}, \hat{t})}}|p| \leqq R_{+}(m),
$$

and moreover Lemma 3.19 (1) and (2) below ensure that

$$
\sup _{\hat{t} \in(0, T)} \sup _{\substack{x, y \in \mathbf{R}^{n} \\ x \neq y}} \frac{|u(x, \hat{t})-u(y, \hat{t})|}{|x-y|} \leqq R_{+}(m) .
$$

We thus conclude that $\operatorname{Lip}_{x}[u] \leqq R_{+}(m)$.

Remark 3.18. In order to prove this proposition in more generality, it is sufficient to assume that $H$ satisfies $\left(\mathrm{H}_{R+}\right)$ or $\left(\mathrm{H}_{R-}\right)$ only for all $m \in I$, where $I$ is the range of $m(\cdot)$ in $\left(\mathrm{H}_{m}\right)$ on $[0, \infty)$. For example $H(x, p)=-|p| /(1+|p|)$ is not coercive but the same conclusion in Proposition 3.17 still holds since we have $0 \leqq m(\rho)<1(\rho \geqq 0)$ and $R_{-}(m)<\infty(0 \leqq m<1)$. 
Lemma 3.19. (1) Let $f: \mathbf{R}^{N} \rightarrow \mathbf{R}$ be bounded. Then we have

$$
\sup _{\substack{x, y \in \mathbf{R}^{N} \\ x \neq y}} \frac{|f(x)-f(y)|}{|x-y|}=\sup _{\substack{\hat{x} \in \mathbf{R}^{N} \\ p \in D^{+} f(\hat{x})}}|p| .
$$

(2) Let $u: Q \rightarrow \mathbf{R}$ be continuous. Assume that $\operatorname{Lip}_{t}[u]<\infty$. Then we have

$$
\sup _{\substack{(\hat{x}, \hat{t}) \in Q \\(p, \tau) \in D^{+} u(\hat{x}, \hat{t})}}|p|=\sup _{\hat{t} \in(0, T)} \sup _{\substack{\hat{x} \in \mathbf{R}^{n} \\ p \in D^{+}\left(\left.u\right|_{t=\hat{t}}\right)(\hat{x})}}|p| .
$$

Proof. (1) Denote by $L_{f}$ and $R_{f}$ respectively the left hand side and the right hand side.

1. We first show $L_{f} \geqq R_{f}$. Fix any $\hat{x} \in \mathbf{R}^{N}, p \in D^{+} f(\hat{x})$ and take a corresponding test function $\phi \in C^{1}\left(\mathbf{R}^{N}\right)$. Since there is some $n_{0} \in S^{n-1}$ such that $|p|=|\nabla \phi(\hat{x})|=\partial \phi / \partial n_{0}(\hat{x})$, we calculate

$$
|p|=\frac{\partial \phi}{\partial n_{0}}(\hat{x})=\lim _{t \downarrow 0} \frac{\phi\left(\hat{x}-t n_{0}\right)-\phi(\hat{x})}{-t} \leqq \lim _{t \downarrow 0} \frac{f\left(\hat{x}-t n_{0}\right)-f(\hat{x})}{-t} \leqq L_{f} .
$$

Here we have used the maximality of $f-\phi$ at $\hat{x}$. This inequality implies $R_{f} \leqq L_{f}$.

2. We next show $L_{f} \leqq R_{f}$. Take any $x^{\prime}, y^{\prime} \in \mathbf{R}^{n}$ such that $x^{\prime} \neq y^{\prime}$. We may assume $f\left(x^{\prime}\right)<f\left(y^{\prime}\right)$. Set $\gamma:=\left(f\left(y^{\prime}\right)-f\left(x^{\prime}\right)\right) /\left|x^{\prime}-y^{\prime}\right|$ and $\phi(x):=\gamma\left|x-x^{\prime}\right|$. Since $f-\phi \rightarrow-\infty$ as $|x| \rightarrow \infty$ by the boundedness of $f$, the function $f-\phi$ attains its maximum at some $\hat{x} \in \mathbf{R}^{N}$. We may let $\hat{x} \neq x^{\prime}$, for otherwise we have $f\left(x^{\prime}\right)=f\left(x^{\prime}\right)-\phi\left(x^{\prime}\right)=f\left(y^{\prime}\right)-\phi\left(y^{\prime}\right)$ and $y^{\prime}$ is another maximizer of $f-\phi$ we can take. Then $\phi$ is $C^{1}$ in some neighborhood of $\hat{x}$ and $\nabla \phi(\hat{x}) \in D^{+} f(\hat{x})$. In view of $|\nabla \phi(\hat{x})|=\gamma$ we have

$$
\frac{f\left(y^{\prime}\right)-f\left(x^{\prime}\right)}{\left|x^{\prime}-y^{\prime}\right|}=\gamma \leqq R_{f},
$$

which yields $L_{f} \leqq R_{f}$.

(2) Denote by $L_{u}$ and $R_{u}$ respectively the left hand side and the right hand side. Then we obtain $L_{u} \leqq R_{u}$ by the separation of variables of a test function. Let us show $L_{u} \geqq R_{u}$. Fix $(\hat{x}, \hat{t}) \in Q, p \in$ $D^{+}\left(\left.u\right|_{t=\hat{t}}\right)(\hat{x})$ and take a corresponding test function $\psi \in C^{1}\left(\mathbf{R}^{n}\right)$. We may assume that $\left.u\right|_{t=\hat{t}}-\psi$ attains its strict maximum at $\hat{x}$. Define $C:=\operatorname{Lip}_{t}[u]+1, g(t):=C|t-\hat{t}|, \phi:=\psi+g, g^{\varepsilon}(t):=C \sqrt{|t-\hat{t}|^{2}+\varepsilon}$ and $\phi^{\varepsilon}:=\psi+g^{\varepsilon}$. Then $u-\phi$ attains its strict maximum at $(\hat{x}, \hat{t})$ and $u-\phi^{\varepsilon}$ converges to $u-\phi$ uniformly. Therefore, by the lemma on convergence of maximum points (see [15, Lemma 2.2.5]), there exists a sequence $\left\{\left(x^{\varepsilon}, t^{\varepsilon}\right)\right\}_{\varepsilon>0}$ such that $\left(x^{\varepsilon}, t^{\varepsilon}\right) \rightarrow(\hat{x}, \hat{t})$ and $u-\phi^{\varepsilon}$ attains its local maximum at $\left(x^{\varepsilon}, t^{\varepsilon}\right)$ for each $\varepsilon>0$. Then we have $\left(\nabla \psi\left(x^{\varepsilon}\right),\left(g^{\varepsilon}\right)^{\prime}\left(t^{\varepsilon}\right)\right) \in D^{+} u^{\varepsilon}\left(x^{\varepsilon}, t^{\varepsilon}\right)$ and $\nabla \psi\left(x^{\varepsilon}\right) \rightarrow \nabla \psi(x)=p$, which yield $L_{u} \geqq R_{u}$.

Proposition 3.20 (BUC preserving property). Assume that $H$ satisfies $\left(\mathrm{H}_{p}\right),\left(\mathrm{H}_{x}\right),\left(\mathrm{H}_{m}\right)$ and $\left(\mathrm{H}_{R+}\right)$. Let $u_{0} \in B U C\left(\mathbf{R}^{n}\right)$ and $u \in S O L\left(H, u_{0}\right)$. Furthermore let $\left\{u_{0}^{\delta}\right\}_{\delta>0} \subset B \operatorname{Bip}\left(\mathbf{R}^{n}\right)$ and assume that $u_{0}^{\delta}$ converges to $u_{0}$ uniformly in $\mathbf{R}^{n}$ as $\delta \downarrow 0$. Then $u \in B U C(Q)$ with modulus of continuity

$$
\omega(r):=\inf _{\delta>0}\left(2\left\|u_{0}-u_{0}^{\delta}\right\|_{\mathbf{R}^{n}}+\sqrt{\left(m^{\delta}\right)^{2}+\left(R_{+}\left(m^{\delta}\right)\right)^{2}} r\right)
$$

where $m^{\delta}:=m\left(\operatorname{Lip}\left[u_{0}^{\delta}\right]\right), m(\cdot)$ is the function in $\left(\mathrm{H}_{m}\right)$ and $R_{+}(\cdot)$ is the function in $\left(\mathrm{H}_{R+}\right)$.

For a given $u_{0} \in B U C\left(\mathbf{R}^{n}\right)$ one can always construct the family $\left\{u_{0}^{\delta}\right\}_{\delta>0}$ like the above by taking $u_{0}^{\delta}$ as the sup- or inf-convolution of $u_{0}$ for $\delta>0$.

In the following proof we use the fact that if uniformly continuous functions $f^{\delta}(\delta>0)$ converges to $f$ uniformly as $\delta \downarrow 0$, then $f$ is also uniformly continuous. Let $\omega^{\delta}$ be a modulus of $f^{\delta}$. Then

$$
\begin{aligned}
|f(x)-f(y)| & \leqq\left|f(x)-f^{\delta}(x)\right|+\left|f^{\delta}(x)-f^{\delta}(y)\right|+\left|f^{\delta}(y)-f(y)\right| \\
& \leqq 2\left\|f-f^{\delta}\right\|+\omega^{\delta}(|x-y|),
\end{aligned}
$$


and hence our claim follows. We also find that $f$ has

$$
\omega(r)=\inf _{\delta>0}\left(2\left\|f-f^{\delta}\right\|+\omega^{\delta}(r)\right)
$$

as its modulus and that there is no need to assume the existence of a common modulus of $f^{\delta}$.

Proof. By the assumption $\left(\mathrm{H}_{m}\right)$ there exists a solution $u^{\delta} \in S O L\left(H, u_{0}^{\delta}\right)$ for each $\delta>0$, and Proposition 3.17 implies that $u^{\delta} \in B \operatorname{Lip}(Q)$ since $u_{0}^{\delta} \in B \operatorname{Lip}\left(\mathbf{R}^{n}\right)$. Now, by using the inequality

$$
\left\|u-u^{\delta}\right\|_{Q} \leqq\left\|u_{0}-u_{0}^{\delta}\right\|_{\mathbf{R}^{n}}
$$

in Remark 2.7 (1) we find that $u^{\delta}$ converges to $u$ uniformly in $Q$ as $\delta \downarrow 0$. Besides, recalling the remark before this proof, we see $u \in B U C(Q)$ and

$$
\omega_{0}(r):=\inf _{\delta>0}\left(2\left\|u-u^{\delta}\right\|_{Q}+\operatorname{Lip}\left[u^{\delta}\right] r\right)
$$

is a modulus of $u$. Applying (3.1) and the estimate of $\operatorname{Lip}\left[u^{\delta}\right]$ in Proposition 3.17, we obtain the desired form of $\omega$.

Since we should treat discontinuous $H$, we apply the above results to the solutions $u^{\varepsilon}$ of the approximate equations and confirm that their infimum has a desired property. We use the fact in Remark 3.15 that if $u^{\varepsilon}$ share a modulus independent of $\varepsilon$, then their infimum has the same modulus. In the case of non-coercive Hamiltonian, solutions cannot preserve even continuity of the initial data as we observed in Example 3.1, in which the envelope solution $u(x, t)=\operatorname{ct} I(x)$ is not continuous in contrast to the initial data $u_{0} \equiv 0$.

Theorem 3.21. Assume that $H$ satisfies $\left(\mathrm{H}_{\varepsilon}\right),\left(\mathrm{H}_{m}\right)$ and that each $H^{\varepsilon}$ in $\left(\mathrm{H}_{\varepsilon}\right)$ satisfies $\left(\mathrm{H}_{m}\right),\left(\mathrm{H}_{R+}\right)$. Assume furthermore that

$$
\sup _{\varepsilon>0} m^{\varepsilon}(\rho)<\infty, \quad \sup _{\varepsilon>0} R_{+}^{\varepsilon}(m)<\infty
$$

for all $\rho \geqq 0$ and $m \geqq 0$, where

$$
\begin{aligned}
m^{\varepsilon}(\rho) & :=\sup \left\{\left|H^{\varepsilon}(x, p)\right| \mid(x, p) \in \mathbf{R}^{n} \times \bar{B}_{\rho}(0)\right\}(<\infty), \\
R_{+}^{\varepsilon}(m) & :=\sup \left\{|p| \mid \exists x \in \mathbf{R}^{n}, H^{\varepsilon}(x, p) \leqq m\right\}(<\infty) .
\end{aligned}
$$

Let $u_{0} \in B U C\left(\mathbf{R}^{n}\right)$. Then $u$, a solution approximated from above, has the following properties.

(1) $u \in B U C(Q)$.

(2) If $u_{0} \in B \operatorname{Lip}\left(\mathbf{R}^{n}\right)$, then $u \in B \operatorname{Lip}(Q)$.

(3) If $H$ satisfies $\left(\mathrm{H}_{p}\right)$, then $u$ is a unique envelope solution of $(\mathrm{HJ})$.

The condition $\left(\mathrm{H}_{R+}\right)$ is able to be replaced by $\left(\mathrm{H}_{R-}\right)$. In this case, if $H$ itself satisfies $\left(\mathrm{H}_{R-}\right)$, then the assumption $\sup _{\varepsilon>0} R_{-}^{\varepsilon}(m)<\infty$ always holds since we have $R_{-}^{\varepsilon}(m) \leqq R_{-}(m)$ by $H^{\varepsilon} \leqq H$.

Proof. We first prove (2) and next show (1) by approximating the initial data. Take $u^{\varepsilon} \in S O L\left(H^{\varepsilon}, u_{0}\right)$ in $\left(\mathrm{H}_{\varepsilon}\right)$.

(2) Denote $l:=\operatorname{Lip}\left[u_{0}\right]$. Now, Proposition 3.17 ensures that $u^{\varepsilon} \in B \operatorname{Lip}(Q)$ and

$$
\operatorname{Lip}_{t}\left[u^{\varepsilon}\right] \leqq m^{\varepsilon}(l) \leqq \sup _{\varepsilon>0} m^{\varepsilon}(l), \quad \operatorname{Lip}_{x}\left[u^{\varepsilon}\right] \leqq R_{+}^{\varepsilon}\left(m^{\varepsilon}(l)\right) \leqq \sup _{\varepsilon>0} R_{+}^{\varepsilon}\left(m^{\varepsilon}(l)\right) .
$$

Since both Lipschitz constants are estimated independently of $\varepsilon$, we conclude $u=\inf _{\varepsilon>0} u^{\varepsilon} \in B L i p(Q)$.

(1) Let $u_{0}^{\delta}=\left(u_{0}\right)^{\delta}$ be the sup-convolution of $u_{0}$ and denote $l^{\delta}:=\operatorname{Lip}\left[u_{0}^{\delta}\right]$. Then, Proposition 3.20 ensures that $u^{\varepsilon} \in B U C(Q)$ and each $u^{\varepsilon}$ has a modulus

$$
\omega^{\varepsilon}(r):=\inf _{\delta>0}\left(2\left\|u_{0}-u_{0}^{\delta}\right\|_{\mathbf{R}^{n}}+\sqrt{\left\{m^{\varepsilon}\left(l^{\delta}\right)\right\}^{2}+\left\{R_{+}^{\varepsilon}\left(m^{\varepsilon}\left(l^{\delta}\right)\right)\right\}^{2}} r\right) .
$$


Since $m^{\varepsilon}\left(l^{\delta}\right)$ and $R_{+}^{\varepsilon}\left(m^{\varepsilon}\left(l^{\delta}\right)\right)$ are similarly estimated independently of $\varepsilon$, there exists a common modulus for $u^{\varepsilon}$. Thus we conclude $u \in B U C(Q)$.

(3) Since $u_{0}^{\delta} \in B \operatorname{Bip}\left(\mathbf{R}^{n}\right)$, there exists a Lipschitz continuous envelope solution $u^{\delta} \in e . S O L\left(H, u_{0}^{\delta}\right) \cap$ $B \operatorname{Lip}(Q)$ for each $\delta>0$ by (2) above. Moreover, there exist solutions of approximate equations $\left(u^{\delta}\right)^{\varepsilon} \in$ $S O L\left(H^{\varepsilon}, u_{0}^{\delta}\right)$, which satisfy

$$
u^{\delta}=\inf _{\varepsilon>0}\left(u^{\delta}\right)^{\varepsilon} \quad \text { and } \quad\left(u^{\delta}\right)^{\varepsilon} \in \bar{D}-S U P\left(H, u_{0}^{\delta}\right) \cap B \operatorname{Lip}(Q) .
$$

Then, by Theorem 2.9 we have $\left\|v-u^{\delta}\right\|_{Q} \leqq\left\|u_{0}-u_{0}^{\delta}\right\|_{\mathbf{R}^{n}}$ for any envelope solution $v$ of (HJ). Hence the uniqueness of $u$ follows because $\lim _{\delta \downarrow 0}\left\|u_{0}-u_{0}^{\delta}\right\|=0$.

We have given some examples of $H$ satisfying $\left(\mathrm{H}_{\varepsilon}\right)$. In Example $3.9 H^{\varepsilon}$ are not coercive because of their boundedness. We therefore impose the coercivity assumption on $H$ in Example 3.10 so as to apply Theorem 3.21 .

Theorem 3.22. Assume that $H$ has the form of (1.9) with $r \in B U S C\left(\mathbf{R}^{n}\right)$. Assume that $H_{0}$ is coercive, uniformly continuous in $\mathbf{R}^{n} \times \mathbf{R}^{n}$ and satisfies $\left(\mathrm{H}_{m}\right)$. Let $u_{0} \in B U C\left(\mathbf{R}^{n}\right)$. Then there exists a unique envelope solution $u$ of $(\mathrm{HJ})$ and it has the following properties.

(1) $u \in B U C(Q)$.

(2) If $u_{0} \in B \operatorname{Lip}\left(\mathbf{R}^{n}\right)$, then $u \in B \operatorname{Bip}(Q)$.

Proof. We assume $\left(\mathrm{H}_{R+}\right)$ because the proof in the case of $\left(\mathrm{H}_{R-}\right)$ is similar. Let $R_{0+}(\cdot)$ be the function in $\left(\mathrm{H}_{R+}\right)$ for $H_{0}$. It is clear that the above $H$ fulfills $\left(\mathrm{H}_{m}\right)$. As observed in Example 3.10, we also learn that $H$ satisfies $\left(\mathrm{H}_{\varepsilon}\right)$ by the approximation $H^{\varepsilon}(x, p)=H_{0}(x, p)-r^{\varepsilon}(x)$, where $r^{\varepsilon}$ is the sup-convolution of $r$. Thus by Proposition 3.7 we obtain a solution approximated from above $u \in e . S O L\left(H, u_{0}\right)$. It remains to show the uniform boundedness of $m^{\varepsilon}(\rho)$ and $R_{+}^{\varepsilon}(m)$ in $\varepsilon$ in order to apply Theorem 3.21. Since $H^{\varepsilon} \leqq\left|H_{0}\right|+\|r\|_{\mathbf{R}^{n}}$, we have $m^{\varepsilon}(\rho) \leqq \max _{\mathbf{R}^{n} \times \bar{B}_{\rho}(0)}\left|H_{0}\right|+\|r\|<\infty$, and hence $\sup _{\varepsilon>0} m^{\varepsilon}(\rho)<\infty$. Also, when $m \geqq H^{\varepsilon}(x, p)$, one observes that $H_{0}(x, p) \leqq m+r^{\varepsilon} \leqq m+\|r\|$ and so $|p| \leqq R_{0+}(m+\|r\|)$ by $\left(\mathrm{H}_{R+}\right)$. Therefore we obtain $R_{+}^{\varepsilon}(m) \leqq R_{0+}(m+\|r\|)<\infty$, which yields $\sup _{\varepsilon>0} R_{+}^{\varepsilon}(m)<\infty$.

\section{Relaxed Hamiltonians}

In this section we establish a unique existence result without the coercivity assumption for $H$. Our existence result (Proposition 3.7) does not require the coercivity. The problem lies in the uniqueness part. In fact, we cannot expect the uniqueness in general as we observed in Example 3.16. However, we are able to show the uniqueness for more restrictive Hamiltonians without the coercivity. To apply our Lipschitz version of CP (Theorem 2.9) we need Lipschitz continuity of one of solutions, but the continuity preserving property does not hold in general without the coercivity. On the other hand, our general version of CP (Theorem 2.6) excludes Hamiltonians with discontinuous source terms. We solve this difficulty by considering a relaxed problem. If an envelope solution $u$ of (HJ) can be regarded as an envelope solution of another problem (relaxed problem):

$$
\text { (r.HJ) } \begin{cases}\partial_{t} u+\hat{H}(x, \nabla u)=0 & \text { in } Q \\ \left.u\right|_{t=0}=u_{0} & \text { in } \mathbf{R}^{n}\end{cases}
$$

with a relaxed Hamiltonian $\hat{H}$ satisfying $\left(\mathrm{H}_{x N}\right)$, then we conclude the uniqueness of $u$ as envelope solutions of (HJ) by Theorem 2.6.

We define the relaxed Hamiltonians so that $\hat{H} \geqq H$. Then it is obvious that a supersolution of (1.1) is also a supersolution of (4.1). Therefore it is an important issue whether or not a subsolution of (1.1) is also a subsolution of (4.1). We will solve this problem after defining $\hat{H}$. In addition, as another topics about $\hat{H}$ we discuss stability and existence of $\bar{D}$-solutions which are not guaranteed for original $H$. 


\subsection{Uniqueness revisited}

In this section we treat special Hamiltonians with the following properties.

$\left(\mathrm{H}_{r}\right) \quad$ (i) $H$ is lower semicontinuous in $\mathbf{R}^{n} \times \mathbf{R}^{n}$ and is continuous in $\left(\mathbf{R}^{n} \backslash \Gamma\right) \times \mathbf{R}^{n}$ for some $\Gamma$ which satisfies the following:

for every $a \in \Gamma$ there exists a open set $V_{a}$ such that $\{a\}=\Gamma \cap V_{a}$.

(ii) $H^{*}$ is continuous in $\mathbf{R}^{n} \times \mathbf{R}^{n}$.

(iii) $H(a, p) \leqq \inf _{0 \leqq \mu \leqq 1} H^{*}(a, \mu p)$ for each $a \in \Gamma$ and $p \in \mathbf{R}^{n}$.

For such $H$, we define a relaxed Hamiltonian $\hat{H}: \mathbf{R}^{n} \times \mathbf{R}^{n} \rightarrow \mathbf{R}$ by

$$
\hat{H}(x, p):= \begin{cases}H(x, p) & (x \notin \Gamma), \\ \min \left\{\inf _{0 \leqq \mu \leqq 1} H^{*}(x, \mu p), \sup _{0 \leqq \mu \leqq 1} H(x, \mu p)\right\} & (x \in \Gamma) .\end{cases}
$$

(See Figure 7.) The continuity of $H^{*}$ implies that

$$
H^{*}(x, p)=\lim _{\substack{(y, q) \rightarrow(x, p) \\ y \neq x}} H(y, q)
$$

for all $(x, p) \in \mathbf{R}^{n} \times \mathbf{R}^{n}$. Also, since $H(x, p) \leqq \sup _{0 \leqq \mu \leqq 1} H(x, \mu p)$ and $\left(\mathrm{H}_{r}\right)($ iii) holds, we have $H \leqq$ $\hat{H}$ in $\mathbf{R}^{n} \times \mathbf{R}^{n}$. Besides, it is seen that $\hat{H} \leqq H^{*}$ in $\mathbf{R}^{n} \times \mathbf{R}^{n}$ and $\hat{H}$ is lower semicontinuous.

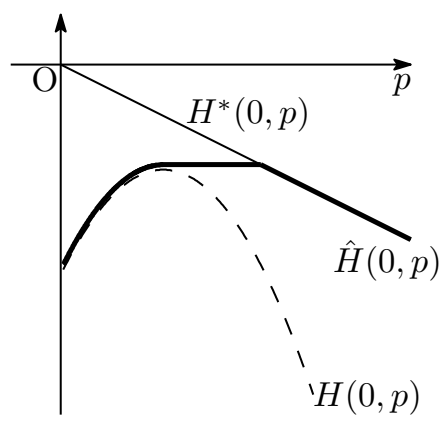

Figure 7: The definition of $\hat{H}(0, p)$ in the case $0 \in \Gamma$.

Example 4.1. Let $H$ have the form of (1.9). Then the following (i)'-(iii)' is one sufficient condition for $\left(\mathrm{H}_{r}\right)$.

(i) ${ }^{\prime} H_{0}$ is continuous in $\mathbf{R}^{n} \times \mathbf{R}^{n}$. $r$ is upper semicontinuous in $\mathbf{R}^{n}$ and is continuous in $\mathbf{R}^{n} \backslash \Gamma$ for some $\Gamma$ which satisfies (4.3).

(ii) ${ }^{\prime} r_{*}$ is continuous in $\mathbf{R}^{n}$.

(iii) $H_{0}(a, p)-r(a) \leqq \inf _{0 \leqq \mu \leqq 1} H_{0}(a, \mu p)-r_{*}(a)$ for each $a \in \Gamma$ and $p \in \mathbf{R}^{n}$.

To show (iii)' it is enough to prove that

$$
\text { a function } \mu \mapsto H_{0}(a, \mu p) \text { is nonincreasing on }\{\mu \geqq 0\} \text { for each } a \in \Gamma \text { and } p \in \mathbf{R}^{n} \text {. }
$$

We here assume (4.5) and let $0 \in \Gamma$ (, i.e., $r$ is discontinuous at 0 ). Then, since $H^{*}(0, \mu p)=H_{0}(0, \mu p)-$ $r_{*}(0)$ and $H(0, \mu p)=H_{0}(0, \mu p)-r(0)$, we have

$$
\hat{H}(0, p)=\min \left\{H_{0}(0, p)-r_{*}(0), H_{0}(0,0)-r(0)\right\}
$$


for all $p \in \mathbf{R}^{n}$. By this equality we find that $\hat{H}(0, p)$ is a constant $H_{0}(0,0)-r(0)=H(0,0)$ on $V:=\left\{p \in \mathbf{R}^{n} \mid H_{0}(0, p) \geqq H_{0}(0,0)-\left(r(0)-r_{*}(0)\right)\right\}$. Furthermore, if $V$ is bounded, namely $V \subset B_{N}(0)$ for some $N>0$, then $\hat{H}(x, p)=H_{0}(x, p)-r_{*}(x)$ holds in $\mathbf{R}^{n} \times\left(\mathbf{R}^{n} \backslash B_{N}(0)\right)$. Therefore assumptions $\left(\mathrm{H}_{p}\right)$ and $\left(\mathrm{H}_{x N}\right)$ required in Theorem 2.6 are fulfilled if $H_{0}$ and $r_{*}$ are uniformly continuous.

Example 4.2. We see for (1.3) that $\hat{H}(x, p)=-|p|-(c I(x)-|p|)_{+}$. As for a unique envelope solution $u(x, t)=c(t-|x|)_{+}$with $u_{0} \equiv 0$, an easy computation shows that $u \in S U B(\hat{H}, 0)$, which implies $u \in e . S O L(\hat{H}, 0)$. Moreover one can also verify $u \in \bar{D}-S O L(\hat{H}, 0)$. This suggests that an envelope solution of (HJ) has a more chance to be a $\bar{D}$-solution of (r.HJ) than the original equation. The details will be discussed in the next subsection.

Example 4.3. For (1.15) we have $\hat{H}=H$. Hence the relaxation method does not give any new information to us.

The following is the key fact for relaxed Hamiltonians.

Lemma 4.4. Assume that $H$ satisfies $\left(\mathrm{H}_{r}\right)$. If $u \in S U B(H)$, then $u \in S U B(\hat{H})$.

Proof. We simply write $u$ for $u^{*}$. Take any $(\hat{x}, \hat{t}) \in Q$ and $(p, \tau) \in D^{+} u(\hat{x}, \hat{t})$. If $\hat{x} \notin \Gamma$ or $p=0$, we deduce $\tau+\hat{H}(\hat{x}, p) \leqq 0$ since $\hat{H}(\hat{x}, p)=H(\hat{x}, p)$ and $u \in S U B(H)$. Therefore we need only consider the case that $\hat{x} \in \Gamma$ and $p \neq 0$. We may assume $\hat{x}=0$ to simplify the notation. Our goal is now to show $\tau+\hat{H}(0, p) \leqq 0$, namely

$$
\tau+\inf _{0 \leqq \mu \leqq 1} H^{*}(0, \mu p) \leqq 0 \quad \text { or } \quad \tau+\sup _{0 \leqq \mu \leqq 1} H(0, \mu p) \leqq 0
$$

Define

$$
\Sigma:=\left\{\mu \in[0,1] \mid(\mu p, \tau) \in D^{+} u(0, \hat{t})\right\}, \quad \mu_{0}:=\inf \{\mu \in[0,1] \mid[\mu, 1] \subset \Sigma\} .
$$

Then $1 \in \Sigma$ and we also have $\mu_{0} \in \Sigma$ since superdifferentials are closed. We discuss two different cases about $\mu_{0}$.

Case 1: $\mu_{0}=0$. Since $(\mu p, \tau) \in D^{+} u(0, \hat{t})$ for each $\mu \in[0,1]$, it follows from $u \in S U B(H)$ that

$$
\tau+H(0, \mu p) \leqq 0 .
$$

Thus we obtain

$$
\tau+\sup _{0 \leqq \mu \leqq 1} H(0, \mu p) \leqq 0
$$

Case 2: $0<\mu_{0} \leqq 1$. Take a corresponding test function $\phi \in C^{1}(Q)$ for $\left(\mu_{0} p, \tau\right) \in D^{+} u(0, \hat{t})$. We may assume $u-\phi$ attains its strict maximum at $(0, \hat{t})$. By the definition of $\mu_{0}$ there exists a sequence $\left\{\mu_{m}\right\}_{m \in \mathbf{N}}$ such that $\mu_{m} \uparrow \mu_{0}$ and $\mu_{m} \notin \Sigma$. Define

$$
\phi_{m}(x, t)=\phi(x, t)-\left(\mu_{0}-\mu_{m}\right)\langle x, p\rangle
$$

for each $m$. Since $\phi_{m}$ converges to $\phi$ locally uniformly, there exists some sequence $\left\{\left(x_{m}, t_{m}\right)\right\}_{m \in \mathbf{N}}$ such that $\left(x_{m}, t_{m}\right) \rightarrow(0, \hat{t})$ and $\max _{Q^{\prime}}\left(u-\phi_{m}\right)=\left(u-\phi_{m}\right)\left(x_{m}, t_{m}\right)$. Here $Q^{\prime}$ is an arbitrary bounded open subset of $Q$ containing $(0, \hat{t})$. The facts that $\mu_{m} \notin \Sigma$ and $\nabla \phi_{m}(0, \hat{t})=\mu_{m} p$ imply $\left(x_{m}, t_{m}\right) \neq(0, \hat{t})$. Moreover we find that $x_{m} \neq 0$ since $\phi_{m}(0, t)=\phi(0, t)$. Thus it follows from $u \in S U B(H)$ that

$$
\partial_{t} \phi\left(x_{m}, t_{m}\right)+H\left(x_{m}, \nabla \phi\left(x_{m}, t_{m}\right)-\left(\mu_{0}-\mu_{m}\right) p\right) \leqq 0
$$

and by letting $m \rightarrow \infty$ we obtain

$$
\tau+H^{*}\left(0, \mu_{0} p\right) \leqq 0
$$

on account of (4.4). As a result we have

$$
\tau+\inf _{0 \leqq \mu \leqq 1} H^{*}(0, \mu p) \leqq 0,
$$

which concludes the proof. 
We present a uniqueness result in a general form.

Proposition 4.5 (uniqueness by relaxation). Assume that $H$ satisfies $\left(\mathrm{H}_{r}\right)$ and that $\hat{H}$ satisfies $\left(\mathrm{H}_{p}\right)$, $\left(\mathrm{H}_{x N}\right)$. Then there exists at most one envelope solution of $(\mathrm{HJ})$ and it is upper semicontinuous.

Proof. If $u_{1}, u_{2} \in e . S O L\left(H, u_{0}\right)$, then $u_{1}, u_{2} \in e . S O L\left(\hat{H}, u_{0}\right)$ by Lemma 4.4 and $H \leqq \hat{H}$. Since $\hat{H}$ satisfies $\left(\mathrm{H}_{p}\right),\left(\mathrm{H}_{x N}\right)$ and is lower semicontinuous, we see $u_{1}=u_{2}$ and they are upper semicontinuous in terms of Proposition 3.6.

Here we give one sufficient condition to apply Proposition 4.5.

Proposition 4.6. Assume that $H$ has the form of (1.9) with (1.11). Assume that $H_{0}$ is uniformly continuous in $\mathbf{R}^{n} \times \mathbf{R}^{n}$, satisfies (4.5) and

$$
R(\gamma):=\sup \left\{|p| \mid \exists x \in \mathbf{R}^{n}, H_{0}(x, p) \geqq-\gamma\right\}<\infty,
$$

where $\gamma:=\max _{j=1}^{N}\left(c_{j}-H_{0}\left(a_{j}, 0\right)\right)$. Then $\hat{H}$ satisfies $\left(\mathrm{H}_{p}\right)$ and $\left(\mathrm{H}_{x N}\right)$.

Proof. According to Example 4.1 we have

$$
\hat{H}(x, p):= \begin{cases}H_{0}(x, p) & \left(x \neq a_{j}\right), \\ \min \left\{H_{0}\left(a_{j}, p\right), H_{0}\left(a_{j}, 0\right)-c_{j}\right\} & \left(x=a_{j}\right)\end{cases}
$$

under the above assumptions. It suffices to check $\left(\mathrm{H}_{x N}\right)$. By (4.6) we see $H_{0}\left(a_{j}, p\right)<H_{0}\left(a_{j}, 0\right)-c_{j}$ for all $p \in \mathbf{R}^{n} \backslash \bar{B}_{R(\gamma)}(0)$ and $j \in\{1,2, \ldots, N\}$. Consequently

$$
\hat{H}(x, p)=H_{0}(x, p) \quad \text { if }(x, p) \in \mathbf{R}^{n} \times\left(\mathbf{R}^{n} \backslash \bar{B}_{R(\gamma)}(0)\right),
$$

and so $\left(\mathrm{H}_{x N}\right)$ is satisfied.

Example 4.7. Let us consider the non-coercive Hamiltonian (1.16). Then (4.6) is fulfilled if and only if $0<c<1$, and therefore the uniqueness of envelope solutions follows. We will later see in Example 5.15 that there are infinitely many $\bar{D}$-solutions even with $u_{0} \equiv 0$ when $c>1$.

Theorem 4.8. Assume that $H$ has the form of (1.16). Let $u_{0} \in B U C\left(\mathbf{R}^{n}\right)$ and $0<c<1$. Then there exists a unique envelope solution $u^{c}$ of $(\mathrm{HJ})$. Moreover $u^{c} \in B L i p(Q)$ provided that $\operatorname{Lip}\left[u_{0}\right]<(1-c) / c$.

Proof. Since $H=H^{c}$ satisfies $\left(\mathrm{H}_{\varepsilon}\right)$ and $\left(\mathrm{H}_{m}\right)$ for Proposition 3.7 (refer to Example 3.9 or 3.10 about $\left.\left(\mathrm{H}_{\varepsilon}\right)\right)$, there exists $u^{c} \in e . S O L\left(H^{c}, u_{0}\right)$, a solution approximated from above, and its uniqueness follows from Example 4.7.

We next show the Lipschitz continuity preserving property of $u^{c}$. Define $H^{c, \varepsilon}(x, p)=-|p| /(1+|p|)-$ $c I^{\varepsilon}(x)$ with (1.7). Observe that for fixed $\rho_{0}>0$

$$
m^{c, \varepsilon}(\rho):=\sup \left\{\left|H^{c, \varepsilon}(x, p)\right| \mid(x, p) \in \mathbf{R}^{n} \times \bar{B}_{\rho}(0)\right\} \leqq c+\frac{\rho_{0}}{1+\rho_{0}} \quad\left(\forall \rho \in\left[0, \rho_{0}\right]\right)
$$

and

$$
R_{-}^{c, \varepsilon}(m):=\sup \left\{|p| \mid \exists x \in \mathbf{R}^{n}, H^{c, \varepsilon}(x, p) \geqq-m\right\} \leqq \frac{m}{1-m} \quad(\forall m \in[0,1)) .
$$

Therefore we learn by Remark 3.18 that solutions of (HJ) with $H^{c, \varepsilon}$ are Lipschitz continuous provided that $c+\rho_{0} /\left(1+\rho_{0}\right)<1$, i.e., $\rho_{0}<(1-c) / c$. Furthermore, we see by the estimate above that their Lipschitz constants are bounded uniformly in $\varepsilon$. Hence their infimum, which is $u^{c}$ by the uniqueness, is also Lipschitz continuous if $\operatorname{Lip}\left[u_{0}\right]<(1-c) / c$.

We think that the Lipschitz continuity preserving property may not hold if $\operatorname{Lip}\left[u_{0}\right]>(1-c) / c$. 


\subsection{Existence of $\bar{D}$-solutions}

For (HJ) with (1.3) and $u_{0} \equiv 0$, the unique envelope solution $u(x, t)=c(t-|x|)_{+}$is not only an envelope solution of (r.HJ) but also a $\bar{D}$-solution of (r.HJ). In other words, we obtained a $\bar{D}$-solution by the relaxation method while our original problem $(\mathrm{HJ})$ has no $\bar{D}$-solution. Unfortunately, for a general initial-value it is not always true that $u \in \bar{D}-S O L\left(\hat{H}, u_{0}\right)$ when $u \in e . S O L\left(H, u_{0}\right)$. Its counterexample is given by the lower right function in Figure 10 later. It is the envelope solution of (HJ) with $H(x, p)=-|p|-I(x), u_{0}(x)=2 \min \{|x|, 1\}$ and is written as

$$
u(x, t)= \begin{cases}2 \min \{|x|+t, 1\} & (t \leqq 1), \\ (t-|x|-1)_{+}+2 & (t \geqq 1) .\end{cases}
$$

For this $u$, we observe that $u \notin \bar{D}-S O L\left(\hat{H}, u_{0}\right)$ because $(0,0) \in \bar{D}^{-} u(0,1)$ and $0+\hat{H}(0,0)=-1<0$. In this subsection we consider what conditions lead an envelope solution of (HJ) to a $\bar{D}$-solution of (r.HJ).

Recall that an envelope supersolution is not always a $\bar{D}$-supersolution because of a lack of stability. If it is guaranteed for (r.HJ), one can obtain a $\bar{D}$-solution. We here think of what is trouble in general about the stability for $(\mathrm{r} . \mathrm{HJ})$. Let $u:=\inf _{\varepsilon>0} u^{\varepsilon}, u^{\varepsilon} \in \bar{D}-S U P(\hat{H}),(p, \tau) \in \bar{D}^{-} u_{*}(\hat{x}, \hat{t})$ and take a defining approximate sequence $\left(p_{m}, \tau_{m}\right) \in D^{-} u_{*}\left(x_{m}, t_{m}\right)$. Since $u^{\varepsilon} \in S U P(\hat{H})$ in particular, the stability for standard solutions ensures $\tau_{m}+(\hat{H})^{*}\left(x_{m}, p_{m}\right) \geqq 0$. Sending $m \rightarrow \infty$, we see $\tau+(\hat{H})^{*}(\hat{x}, p) \geqq 0$ and $\tau+\hat{H}(\hat{x}, p) \geqq 0$ if $\hat{H}$ is continuous at $(\hat{x}, p)$. Hence the remaining problem is whether $\tau+\hat{H}(\hat{x}, p) \geqq 0$ holds for every $(p, \tau) \in \bar{D}^{-} u_{*}(\hat{x}, \hat{t})$ such that $(\hat{x}, p)$ is a discontinuous point of $\hat{H}$.

Let us come back to the example of (1.3). Since the set of discontinuous points of $\hat{H}(x, p)=$ $-|p|-(c I(x)-|p|)_{+}$is $\{(0, p)|| p \mid<c\}$, the problem is whether $\tau-c \geqq 0$ holds for all $(p, \tau) \in \bar{D}^{-} u_{*}(0, \hat{t})$ such that $|p|<c$. This can be regarded as a condition about growth rates of $u$ in the $t$-direction near $\{0\} \times(0, T)$ and is satisfied for example if $u$ has the form $u(x, t)=c(t-|x|)_{+}+k$ for some $k \in \mathbf{R}$. According to Example 5.7 later, if the initial-value $u_{0}$ satisfies $u_{0}(x)<c|x|+u_{0}(0)$ for all $x \in \mathbf{R}^{n} \backslash\{0\}$, then $u(x, t)=c(t-|x|)_{+}+u_{0}(0)$ near $\{0\} \times(0, T)$. Thus we obtain a $\bar{D}$-solution of $(\mathrm{r} . \mathrm{HJ})$.

Proposition 4.9. Let $H(x, p)=-|p|-c I(x)$ for $c>0$. Let $u \in e . S O L\left(H, u_{0}\right)$. If $u_{0}(x)<c|x|+u_{0}(0)$ for all $x \in \mathbf{R}^{n} \backslash\{0\}$, then $u \in \bar{D}-S O L\left(\hat{H}, u_{0}\right)$.

In order to treat more Hamiltonians, we impose additional assumptions on $H$ satisfying $\left(\mathrm{H}_{r}\right)$. For convenience we restrict discontinuous points of $H$.

$\left(\mathrm{H}_{c}\right) \quad$ (i) $H$ satisfies $\left(\mathrm{H}_{r}\right)$ with $\Gamma=\{0\}$.

(ii) There exists a constant $c_{H}$ such that $c_{H}=\sup _{0 \leqq \mu \leqq 1} H(0, \mu p)$ for all $p \in \mathbf{R}^{n}$.

(iii) $\inf _{0 \leqq \mu \leqq 1} H^{*}(0, \mu p)=H^{*}(0, p)$ for all $p \in P:=\left\{p \in \mathbf{R}^{n} \mid \inf _{0 \leqq \mu \leqq 1} H^{*}(0, \mu p)<c_{H}\right\}$.

When $H$ fulfills $\left(\mathrm{H}_{c}\right)$, we have

$$
\hat{H}(0, p)= \begin{cases}c_{H} & (p \notin P), \\ H^{*}(0, p) & (p \in P) .\end{cases}
$$

Note that $P$ is an open set by the continuity of $H^{*}$ and that $\hat{H}$ is continuous in $\{(x, p) \mid x \neq 0$ or $p \in P\}$. We here introduce a new notion of supersolutions for which one can easily show stability.

Definition 4.10 (singular supersolutions). Let $H$ satisfy $\left(\mathrm{H}_{c}\right)$. A function $u: Q \rightarrow \mathbf{R}$ is called a singular supersolution of (4.1) if it is bounded from below, is continuous on $\{0\} \times(0, T)$ and satisfies the following:

(\#1) $\tau+c_{H} \geqq 0$ for all $\hat{t} \in(0, T)$ and $\tau \in D^{-}\left(\left.u\right|_{x=0}\right)(\hat{t})$.

$(\# 2) \tau+\hat{H}(\hat{x}, p) \geqq 0$ for all $(p, \tau) \in D^{-} u_{*}(\hat{x}, \hat{t})$ satisfying $\hat{x} \neq 0$ or $p \in P$.

We denote by $\operatorname{SUP}^{\#}(\hat{H})$ the set which consists of all singular supersolutions of (4.1). 
By virtue of the definition above, the standard argument of the stability for supersolutions applies.

Proposition 4.11 (stability for singular supersolutions). Assume that $H$ satisfies $\left(\mathrm{H}_{c}\right)$. Let $\mathcal{S}$ be a nonempty subset of $S U P^{\#}(\hat{H})$. If $u:=\inf _{w \in \mathcal{S}} w$ is bounded from below and is continuous on $\{0\} \times(0, T)$, then $u \in S U P^{\#}(\hat{H})$.

Since our original goal is the stability for $\bar{D}$-supersolutions, we next show equivalence between $\bar{D}$ supersolutions and singular supersolutions under suitable assumptions.

Lemma 4.12. Assume that $H$ satisfies $\left(\mathrm{H}_{c}\right)$. Let $u: Q \rightarrow \mathbf{R}$ be Lipschitz continuous in $Q$ and satisfy the following.

(U1) For all $\hat{t} \in(0, T)$ there exist some $d>0, \delta>0$ and $C>0$ such that $\left.u\right|_{x=x^{\prime}}+C t^{2}$ is convex in $(\hat{t}-\delta, \hat{t}+\delta)$ for each $x^{\prime} \in B_{d}(0)$.

Then $u \in \bar{D}-S U P(\hat{H})$ if and only if $u \in S U P^{\#}(\hat{H})$.

Remark 4.13. (1) The condition (U1) means a some kind of semiconvexity of $u$ with respect to $t$. Here we say a function $f$ is semiconvex if there exists a constant $C>0$ such that $f(x)+C|x|^{2}$ is convex. When $f(x)+C|x|^{2}$ is convex, functions $f(x)+C|x-a|^{2}$ are also convex for all $a \in \mathbf{R}^{n}$. This claim is confirmed by seeing $f(x)+C|x-a|^{2}=f(x)+C|x|^{2}-2 C\langle x, a\rangle+C|a|^{2}$ since the right hand side is the sum of two convex functions.

(2) If we do not assume (U1), the singular supersolutions are not necessarily $\bar{D}$-supersolutions. Indeed, $u$ defined by (4.7) is not a $\bar{D}$-supersolution but a singular supersolution of (r.HJ), where $H(x, p)=$ $-|p|-I(x)$ and $u_{0}(x)=2 \min \{|x|, 1\}$. It does not satisfy (U1) at $\hat{t}=1$.

Proof. 1. We first show that $u$ satisfying (U1) has the following property.

$$
\tau \in D^{-}\left(\left.u\right|_{x=0}\right)(\hat{t}) \quad \text { for all } \hat{t} \in(0, T) \text { and }(p, \tau) \in \bar{D}^{-} u(0, \hat{t})
$$

Let $\hat{t} \in(0, T),(p, \tau) \in \bar{D}^{-} u(0, \hat{t})$ and take a defining approximate sequence $\left(p_{m}, \tau_{m}\right) \in D^{-} u\left(x_{m}, t_{m}\right)$ and their corresponding test functions $\phi_{m} \in C^{1}(Q)$. We may assume $\phi_{m}(x, t)=\psi_{m}(x)+g_{m}(t)$ for some $\psi_{m} \in C^{1}\left(\mathbf{R}^{n}\right)$ and $g_{m} \in C^{1}(0, T)$. Since $\left.u\right|_{x=x_{m}}-g_{m}$ attains its minimum at $t_{m}$, the function $\left.u\right|_{x=x_{m}}+C\left(t-t_{m}\right)^{2}-g_{m}$ also attains its minimum at $t_{m}$, where $C$ is the constant in (U1). Then $\left.u\right|_{x=x_{m}}+C\left(t-t_{m}\right)^{2}$ is convex in $(\hat{t}-\delta, \hat{t}+\delta)$ and so we may assume $g_{m}$ is linear, i.e., $g_{m}(t)=L_{m} t$ for some $L_{m} \in \mathbf{R}$. We find that $L_{m}=g_{m}^{\prime}\left(t_{m}\right)=\tau_{m} \rightarrow \tau$ as $m \rightarrow \infty$. Next, define $\bar{g}(t):=\tau t$. Then it is easily seen that $\left.u\right|_{x=0}+C(t-\hat{t})^{2}-\bar{g}=\left.u\right|_{x=0}-\left\{\bar{g}-C(t-\hat{t})^{2}\right\}$ attains its local minimum at $\hat{t}$ by the continuity of $u$ at $(0, \hat{t})$. Thus we deduce $\tau \in D^{-}\left(\left.u\right|_{x=0}\right)(\hat{t})$ since $(d / d t)\left\{\bar{g}-C(t-\hat{t})^{2}\right\}(\hat{t})=\tau$.

2. We prove that a $\bar{D}$-supersolution $u$ of (4.1) is a singular supersolution. It is easy to confirm (\#2), and so we only need to show $(\# 1)$. Fix $\hat{t} \in(0, T), \tau \in D^{-}\left(\left.u\right|_{x=0}\right)(\hat{t})$ and take a corresponding test function $g \in C^{1}(0, T)$. We may assume $\left.u\right|_{x=0}-g$ attains its strict minimum at $\hat{t}$. Define $\gamma:=\operatorname{Lip}_{x}[u]+1$, $\psi(x):=-\gamma|x|, \phi:=\psi+g, \psi^{\varepsilon}(x):=-\gamma \sqrt{|x|^{2}+\varepsilon}$ and $\phi^{\varepsilon}:=\psi^{\varepsilon}+g$. Then $u-\phi$ attains its strict minimum at $(0, \hat{t})$ and $u-\phi^{\varepsilon}$ converges to $u-\phi$ uniformly. Therefore there exists a sequence $\left\{\left(x^{\varepsilon}, t^{\varepsilon}\right)\right\}_{\varepsilon>0}$ such that $\left(x^{\varepsilon}, t^{\varepsilon}\right) \rightarrow(0, \hat{t})$ as $\varepsilon \downarrow 0$ and $u-\phi^{\varepsilon}$ attains its local minimum at $\left(x^{\varepsilon}, t^{\varepsilon}\right)$ for each $\varepsilon>0$. Now, since $\left|\nabla \psi^{\varepsilon}\right| \leqq \gamma$ for all $\varepsilon>0$, we may let $\nabla \psi^{\varepsilon}\left(x^{\varepsilon}\right)$ converge to some $\bar{p} \in \mathbf{R}^{n}$. Since we also have $g^{\prime}\left(t^{\varepsilon}\right) \rightarrow g^{\prime}(\hat{t})=\tau$ as $\varepsilon \downarrow 0$, we obtain $(\bar{p}, \tau) \in \bar{D}^{-} u(0, \hat{t})$. Thus it follows from $u \in \bar{D}-S U P(\hat{H})$ that $0 \leqq \tau+\hat{H}(0, \bar{p}) \leqq \tau+c_{H}$.

3. We next show that a singular supersolution $u$ of $(4.1)$ is a $\bar{D}$-supersolution. Fix $(p, \tau) \in \bar{D}^{-} u(\hat{x}, \hat{t})$. We first consider the case that $\hat{x} \neq 0$ or $p \in P$. Take a defining approximate sequence $\left(p_{m}, \tau_{m}\right) \in$ $D^{-} u\left(x_{m}, t_{m}\right)$. According to $(\# 2)$ we have $\tau_{m}+\hat{H}\left(x_{m}, p_{m}\right) \geqq 0$ for very large $m \in \mathbf{N}$. Sending $m \rightarrow \infty$, we obtain $\tau+\hat{H}(\hat{x}, p) \geqq 0$. If $\hat{x}=0$ and $p \notin P$, we have $\tau \in D^{-}\left(\left.u\right|_{x=0}\right)(\hat{t})$ by (4.8). Hence $\tau+c_{H} \geqq 0$ in virtue of $(\# 1)$.

We sum up the above results for the initial-value problem.

Proposition 4.14 (existence of $\bar{D}$-solutions for relaxed problems). Assume that $H$ satisfies $\left(\mathrm{H}_{c}\right)$. Let $u \in \operatorname{e.SOL}\left(H, u_{0}\right) \cap \operatorname{BLip}(Q)$. If $u$ satisfies (U1), then $u \in \bar{D}-S O L\left(\hat{H}, u_{0}\right)$. 
Remark 4.15. The Lipschitz continuity of $u$ is used to show the only-if-part (Step 2) in the proof of Lemma 4.12. When $u$ is not Lipschitz continuous in $Q$, we take $\psi$ and $\psi^{\varepsilon}$ in the proof as

$$
\psi(x)=\left\{\begin{array}{ll}
0 & (x=0) \\
-\infty & (x \neq 0)
\end{array}, \quad \psi^{\varepsilon}(x)=-\frac{|x|^{2}}{\varepsilon} .\right.
$$

Indeed, because $\lim \sup _{\varepsilon \downarrow 0}^{*} \psi^{\varepsilon}=\psi$, there exists a similar sequence $\left\{\left(x^{\varepsilon}, t^{\varepsilon}\right)\right\}_{\varepsilon>0}$ by taking subsequence if necessary. However, since $p^{\varepsilon}:=\nabla \psi^{\varepsilon}\left(x^{\varepsilon}\right)=-2 x^{\varepsilon} / \varepsilon$ in this case, the sequence $\left\{p^{\varepsilon}\right\}_{\varepsilon>0}$ may be unbounded. To deal with such case, it is sufficient to assume

$$
\limsup _{(x, p) \rightarrow(0, \infty)} H(x, p) \leqq c_{H} .
$$

Let $\left|p^{\varepsilon}\right| \rightarrow \infty$ in order to confirm this claim. Note that $x^{\varepsilon} \neq 0$ for very small $\varepsilon$. Since $\left(p^{\varepsilon}, g^{\prime}\left(t^{\varepsilon}\right)\right) \in$ $D^{-} u_{*}\left(x^{\varepsilon}, t^{\varepsilon}\right)$ and $u \in D-S U P(\hat{H})$, we have $g^{\prime}\left(t^{\varepsilon}\right)+H\left(x^{\varepsilon}, p^{\varepsilon}\right) \geqq 0$. Taking lim sup $\mathcal{\varepsilon}_{\varepsilon \downarrow}^{*}$ in the inequality, we obtain $\tau+c_{H} \geqq 0$ by (4.9).

\section{Some examples of solutions}

\subsection{Representaion by optimal control theory}

Let us recall the representation formula of viscosity solutions by optimal control theory. (See for instance [14].) We consider the following state equation.

$$
X^{\prime}(s)=f(X(s), \alpha(s)) \quad \text { in }(0, t), \quad X(0)=x .
$$

Here the unknown is $X:[0, t] \rightarrow \mathbf{R}^{n}$ and

- $x \in \mathbf{R}^{n}$ is a given initial state and $t \in[0, T]$ is a terminal time.

- $A \subset \mathbf{R}^{m}$ is a compact control set and $\alpha \in \mathcal{A}:=\{\alpha:[0, T] \rightarrow A$, measurable $\}$ is a control.

- $f=f(x, a): \mathbf{R}^{n} \times A \rightarrow \mathbf{R}^{n}$ is a given bounded and continuous function. Moreover $f(x, a)$ is Lipschitz continuous in $x$ uniformly in $a$, that is $\operatorname{Lip}_{x}[f]<\infty$.

As for this ODE there exists for each $\alpha \in \mathcal{A}$ a unique Lipschitz continuous solution $X(s)$ which satisfies the first equation of (5.1) a.e. $s \in(0, t)$. Let us write $X(s)=X^{\alpha}(s)=X(s ; \alpha, x, t)$ to denote the solution. Since $\left|X^{\alpha}\left(s_{1}\right)-X^{\alpha}\left(s_{2}\right)\right|=\left|\int_{s_{2}}^{s_{1}} f\left(X^{\alpha}(s), \alpha(s)\right) d s\right| \leqq\|f\|_{\mathbf{R}^{n} \times A} \cdot\left|s_{1}-s_{2}\right|$ for each $\alpha \in \mathcal{A}$, the solutions $X^{\alpha}$ are Lipschitz continuous uniformly in $\alpha$ with the Lipschitz constant smaller than $\|f\|$.

Next, for given $(x, t) \in \mathbf{R}^{n} \times[0, T]$ and $\alpha \in \mathcal{A}$ we define a corresponding cost functional $C_{x, t}[\alpha]$ by

$$
C_{x, t}[\alpha]:=\int_{0}^{t} r\left(X^{\alpha}(s), \alpha(s)\right) d s+u_{0}\left(X^{\alpha}(t)\right)
$$

where

- $r=r(x, a): \mathbf{R}^{n} \times A \rightarrow \mathbf{R}$ is a given bounded and continuous function. Moreover $r(x, a)$ is Lipschitz continuous in $x$ uniformly in $a$, that is $\operatorname{Lip}_{x}[r]<\infty$.

- $u_{0} \in B U C\left(\mathbf{R}^{n}\right)$.

We call the above $r$ a running cost function while $u_{0}$ serves as a terminal cost function. Then the value function $u: \mathbf{R}^{n} \times[0, T] \rightarrow \mathbf{R}$ is defined by

$$
u(x, t):=\sup _{\alpha \in \mathcal{A}} C_{x, t}[\alpha] .
$$

We are able to prove that $u$ is a viscosity solution of a Hamilton-Jacobi-Bellman equation. 
Theorem 5.1 (a PDE for the value function). Let $u$ be defined as above. Then $u$ is a unique viscosity solution of the initial-value problem

$$
\text { (HJB) } \begin{cases}\partial_{t} u-\max _{a \in A}\{\langle f(x, a), \nabla u\rangle+r(x, a)\}=0 & \text { in } Q, \\ \left.u\right|_{t=0}=u_{0} & \text { in } \mathbf{R}^{n} .\end{cases}
$$

Remark 5.2. When the value function is defined as the infimum of costs, namely

$$
u(x, t):=\inf _{\alpha \in \mathcal{A}} C_{x, t}[\alpha],
$$

$u$ becomes a solution of the same equation as above except that the max is replaced by min.

Our goal is to extend the classical theory above for discontinuous equations. Now we study Hamiltonians written by the form $H(x, p)=-\max _{a \in A}\langle f(x, a), p\rangle-r(x)$ with $r \in B U S C\left(\mathbf{R}^{n}\right)$. We hereafter assume that running costs are independent of the control variable $a$. Recall that as Example 3.2 and Proposition 3.7 we are able to construct an envelope solution by regularizing $r$ from above to get $r^{\varepsilon}$ (the sup-convolution method enables us to do that) and taking the infimum of solutions of the approximate problems. That means we take

$$
\begin{aligned}
u^{\varepsilon}(x, t) & :=\sup _{\alpha \in \mathcal{A}} C_{x, t}^{\varepsilon}[\alpha] \quad \text { with } C_{x, t}^{\varepsilon}[\alpha]=\int_{0}^{t} r^{\varepsilon}\left(X^{\alpha}(s)\right) d s+u_{0}\left(X^{\alpha}(t)\right), \\
u(x, t) & :=\lim _{\varepsilon \downarrow 0} u^{\varepsilon}(x, t)=\inf _{\varepsilon>0} u^{\varepsilon}(x, t),
\end{aligned}
$$

and prove that $u$ is an envelope solution. On the other hand, since upper semicontinuous functions are integrable, it is possible to define a cost and value function for our original $r$ which is not necessarily continuous, that is

$$
v(x, t):=\sup _{\alpha \in \mathcal{A}} C_{x, t}[\alpha] \quad \text { with } C_{x, t}[\alpha]=\int_{0}^{t} r\left(X^{\alpha}(s)\right) d s+u_{0}\left(X^{\alpha}(t)\right) .
$$

What is the relationship between this $v$ of the discontinuous problem and $u$ via the approximation (5.7)? Since problems including no $\varepsilon$-perturbation can be directly handled, if $u=v$, it characterizes the limit of $u^{\varepsilon}$.

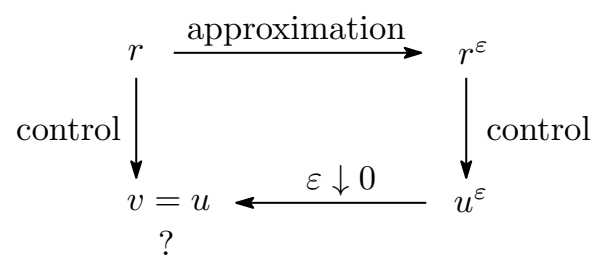

Figure 8: Control theory for a discontinuous running cost. Is this diagram commutative?

In Example 3.2 we deduced that $u(x, t)=\lim _{\varepsilon \downarrow 0} u^{\varepsilon}(x, t)=c(t-|x|)+$ by regarding the Hamiltonian as $H(x, p)=-\max _{a \in \bar{B}_{1}(0)}\langle a, p\rangle-c I(x)$. On the other hand, as for discontinuous case $v(x, t)=$ $\sup _{\alpha \in \mathcal{A}} \int_{0}^{t} c I\left(X^{\alpha}(s)\right) d s$, for each $x \in \mathbf{R}^{n}$ the optimal control is still the one that leads to a straight trajectory before it comes to the origin and stays there after that moment. Therefore we conclude that $v(x, t)=c(t-|x|)_{+}$, and so $u=v$. However, situations are different for another compact set $A$. For example if the control set $A^{\prime}$ is taken as $S^{n-1}$, the resulting Hamiltonian is the same as $H(x, p)=-\max _{a \in S^{n-1}}\langle a, p\rangle-c I(x)$. However, since $X^{\alpha}$ moves at a velocity of 1 all the time for each control $\alpha$, it cannot stay at the origin. Hence we conclude that $v \equiv 0$.

We here give one sufficient condition for guaranteeing $u=v$.

Lemma 5.3 (controllability). Let $r \in B U S C\left(\mathbf{R}^{n}\right), r^{\varepsilon} \in B \operatorname{Lip}\left(\mathbf{R}^{n}\right)(\varepsilon>0)$ and $r^{\varepsilon} \downarrow r$ in $\mathbf{R}^{n}$ pointwise, and define $u^{\varepsilon}$, $u$ and $v$ by (5.6)-(5.8). Assume furthermore that 
(A1) there exists a measurable function $\theta: \mathbf{R}^{n} \times \bar{B}_{\|f\|}(0) \rightarrow A$ such that $p=f(x, \theta(x, p))$ for all $(x, p) \in \mathbf{R}^{n} \times \bar{B}_{\|f\|}(0)$,

where $\|f\|=\sup _{\mathbf{R}^{n} \times A}|f|$. Then $u=v$.

Proof. 1. We find that $v \leqq u$ because $C_{x, t}[\cdot] \leqq C_{x, t}^{\varepsilon}[\cdot]$ for all $\varepsilon>0$. It remains to prove $u \leqq v$. Fix $(x, t) \in$ $Q$. For each $\varepsilon>0$ there is some $\alpha^{\varepsilon} \in \mathcal{A}$ such that $u^{\varepsilon}(x, t)-\varepsilon \leqq C_{x, t}^{\varepsilon}\left[\alpha^{\varepsilon}\right]$. Set $X^{\varepsilon}(s):=X\left(s ; \alpha^{\varepsilon}, x, t\right)$, then one can easily check that the family $\left\{X^{\varepsilon}\right\}_{\varepsilon>0} \subset C[0, t]$ is equicontinuous and uniformly bounded by using Lipschitz continuities of $X^{\alpha}$. Consequently Ascoli-Arzelà theorem ensures that there exists a subseqence $\left\{X^{\varepsilon(j)}\right\}_{j \in \mathbf{N}}$ such that $X^{\varepsilon(j)}$ uniformly converges to some $\bar{X} \in C[0, t]$ as $j \rightarrow \infty$. The estimate $\operatorname{Lip}\left[X^{\alpha}\right] \leqq\|f\|(\forall \alpha)$ implies $\operatorname{Lip}[\bar{X}] \leqq\|f\|$, and so $\bar{X}$ is a.e. differentiable and $\bar{X}^{\prime}(s) \in \bar{B}_{\|f\|}(0)$. Therefore by setting $\bar{\alpha}(s):=\theta\left(\bar{X}(s), \bar{X}^{\prime}(s)\right)$ we have $\bar{X}^{\prime}(s)=f(\bar{X}(s), \bar{\alpha}(s))$ a.e. $s$, and also $\bar{X}(s)=$ $X(s ; \bar{\alpha}, x, t)$.

2. Fix $d>0$. If $0<\varepsilon(j)<d$, then we have

$$
u^{\varepsilon(j)}(x, t) \leqq \varepsilon(j)+C_{x, t}^{\varepsilon(j)}\left[\alpha^{\varepsilon(j)}\right] \leqq \varepsilon(j)+C_{x, t}^{d}\left[\alpha^{\varepsilon(j)}\right]
$$

and

$$
\lim _{j \rightarrow \infty} C_{x, t}^{d}\left[\alpha^{\varepsilon(j)}\right]=\lim _{j \rightarrow \infty}\left\{\int_{0}^{t} r^{d}\left(X^{\varepsilon(j)}(s)\right) d s+u_{0}\left(X^{\varepsilon(j)}(t)\right)\right\}=\int_{0}^{t} r^{d}(\bar{X}(s)) d s+u_{0}(\bar{X}(t)) .
$$

Hence it follows that

$$
u(x, t) \leqq \int_{0}^{t} r^{d}(\bar{X}(s)) d s+u_{0}(\bar{X}(t)) .
$$

Sending $d \downarrow 0$, we obtain by monotone convergence theorem

$$
u(x, t) \leqq \int_{0}^{t} r(\bar{X}(s)) d s+u_{0}(\bar{X}(t))=C_{x, t}[\bar{\alpha}] \leqq v(x, t),
$$

which completes the proof.

Remark 5.4. To prove $u=v$ in the case that value functions are defined by (5.5), there is no need to assume (A1). It suffices to show $u \leqq v$ again. Take a minimizing sequence $\left\{\alpha_{m}\right\}$ of $v(x, t)$ (, i.e., $\left.\lim _{m \rightarrow \infty} C_{x, t}\left[\alpha_{m}\right]=v(x, t)\right)$, and then $u^{\varepsilon}(x, t) \leqq C_{x, t}^{\varepsilon}\left[\alpha_{m}\right]$ holds for all $\varepsilon$ and $m$. Letting $\varepsilon \downarrow 0$, we see that $u(x, t) \leqq C_{x, t}\left[\alpha_{m}\right]$ by monotone convergence theorem. Finally send $m \rightarrow \infty$.

Let us calculate some examples of solutions by applying Lemma 5.3 or Remark 5.4.

At first we consider the case that $H(x, p)=-|p|-r(x)=-\max _{a \in \bar{B}_{1}(0)}\langle a, p\rangle-r(x)$ with $r \in$ $B U S C\left(\mathbf{R}^{n}\right)$. Then (A1) is satisfied by taking $\theta(x, p)=p$, and so Lemma 5.3 guarantees that $v$ defined by

$$
v(x, t):=\sup _{\alpha \in \mathcal{A}}\left\{\int_{0}^{t} r\left(X^{\alpha}(s)\right) d s+u_{0}\left(X^{\alpha}(t)\right)\right\}
$$

is the unique envelope solution.

Example 5.5. Let us consider the case of (1.13) and $u_{0} \equiv 0$. In this case, for each $x \in \mathbf{R}^{n}$ the optimal control forces the state to move straight towards the nearest point in $S$ and to stop moving after the arrival. Therefore we conclude that

$$
v(x, t)=c(t-\operatorname{dist}(x, S))_{+} .
$$

The solution in the case that $S=[-1,1]$ and $c=1$ is given in Figure 9 (the left).

Example 5.6. Let us consider the case of (1.11) and $u_{0} \equiv 0$. In this case, since we have obtained the optimal control for the case $r(x)=c_{j} I\left(x-a_{j}\right)$ for every $j(=1,2, \ldots, N)$, we only need to pick up the maximum of them. Hence we have

$$
v(x, t)=\max _{j=1}^{N} c_{j}\left(t-\left|x-a_{j}\right|\right)_{+} .
$$

The solution in the case that $a_{1}=1, v_{1}=1, a_{2}=-1, v_{2}=1 / 3$ is given in Figure 9 (the right). 

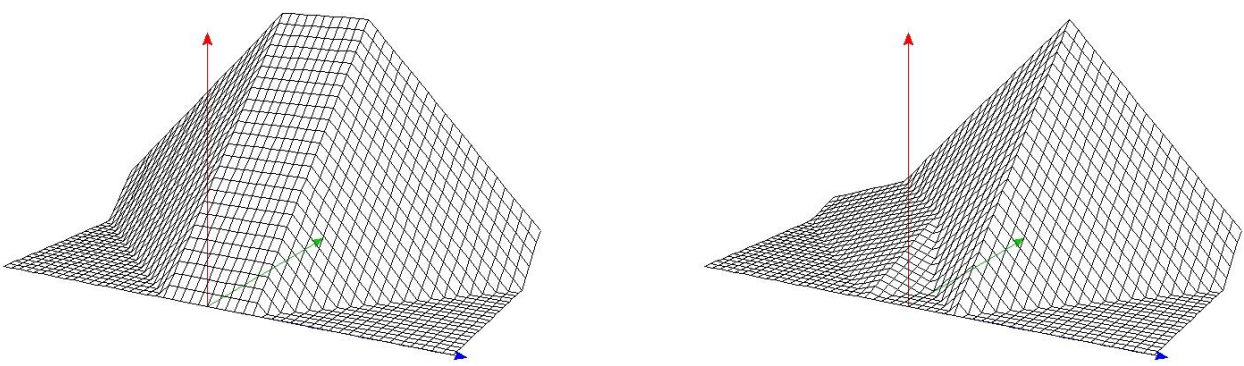

Figure 9: The envelope solution of $\partial_{t} u-|\nabla u|=\chi_{[-1,1]}(x), u_{0} \equiv 0$ (the left) and that of $\partial_{t} u-|\nabla u|=$ $I(x+1) / 3+I(x-1), u_{0} \equiv 0$ (the right).

Example 5.7. Let us consider the case that $r(x)=c I(x)(c>0)$ with a general initial condition $u_{0} \in B U C\left(\mathbf{R}^{n}\right)$. In this case, for each $x \in \mathbf{R}^{n}$ all of the controls can be categorized into two types. One type is to force the state to approach the origin. The other type results in trajectories without passing the origin. The optimal value for the former type is

$$
\max _{s \in[0, t-|x|]}\left\{c s+\max _{\bar{B}_{t-|x|-s}(0)} u_{0}\right\}=: V(x, t)
$$

provided that $t \geqq|x|$ while

$$
\frac{\max }{\bar{B}_{t}(x)} u_{0}
$$

is the maximal value for the latter type. Thus we conclude that

$$
v(x, t)= \begin{cases}\max _{\bar{B}_{t}(x)} u_{0} & (t \leqq|x|), \\ \max \left[\max _{\bar{B}_{t}(x)} u_{0}, V(x, t)\right] & (t \geqq|x|) .\end{cases}
$$

We will make this formula simpler by imposing some conditions on $u_{0}$. Assume that $u_{0}(0)=0$ hereafter.

[1] The case that $u_{0}(x) \leqq c|x|$ in $\mathbf{R}^{n}$. Since $V(x, t)=c(t-|x|)(s=t-|x|)$, we have

$$
v(x, t)= \begin{cases}\max _{\bar{B}_{t}(x)} u_{0} & (t \leqq|x|), \\ \max \left[\max _{\bar{B}_{t}(x)} u_{0}, c(t-|x|)\right] & (t \geqq|x|) .\end{cases}
$$

In particular, we see $u(0, t)=c t$ for all $t \in(0, T)$ because $\max _{\bar{B}_{t}(0)} u_{0} \leqq c t$.

(a) If $u_{0}(x) \leqq 0$ in $\mathbf{R}^{n}$, then $v(x, t)=c(t-|x|)$ for $t \geqq|x|$. The solution for $c=1$ and $u_{0}(x)=-|x| /(1+|x|)$ is given in Figure 10 (the upper left).

(b) If $u_{0}(x)<c|x|$ in $\mathbf{R}^{n} \backslash\{0\}$, then for all $\hat{t} \in(0, T)$ we have $v(x, t)=c(t-|x|)$ in some open neighborhood of $(0, \hat{t}) \in Q$ because $\max _{\bar{B}_{t}(0)} u_{0}<c t$. The solution for $c=1$ and $u_{0}(x)=|x| /(1+|x|)$ is given in Figure 10 (the upper right).

(c) If there is some $\hat{x} \neq 0$ such that $u_{0}(\hat{x})=c|\hat{x}|$, it is unable to take the open neighborhood described in (b) at $(0,|\hat{x}|)$. The solution for $c=1$ and $u_{0}(x)=2 \min \left\{(|x|-1)_{+}, 1\right\}$ is given in Figure 10 (the lower left), where $\hat{x}=2$.

[2] The case that $u_{0}(x) \not c|x|$ in $\mathbf{R}^{n}$. We assume that $u_{0}$ has the form $u_{0}(x)=b(|x|)$ and that $b\left(\rho_{2}\right)-b\left(\rho_{1}\right)>c\left(\rho_{2}-\rho_{1}\right)\left(0 \leqq \rho_{1}<\rho_{2} \leqq R\right), b(\rho)=b(R)(\rho \geqq R)$ for some $R>0$. Note that we have $\max _{\bar{B}_{t}(x)} u_{0}=b(|x|+t)$. Then we observe that $V(x, t)=b(t-|x|-s)(s=0)$ for 

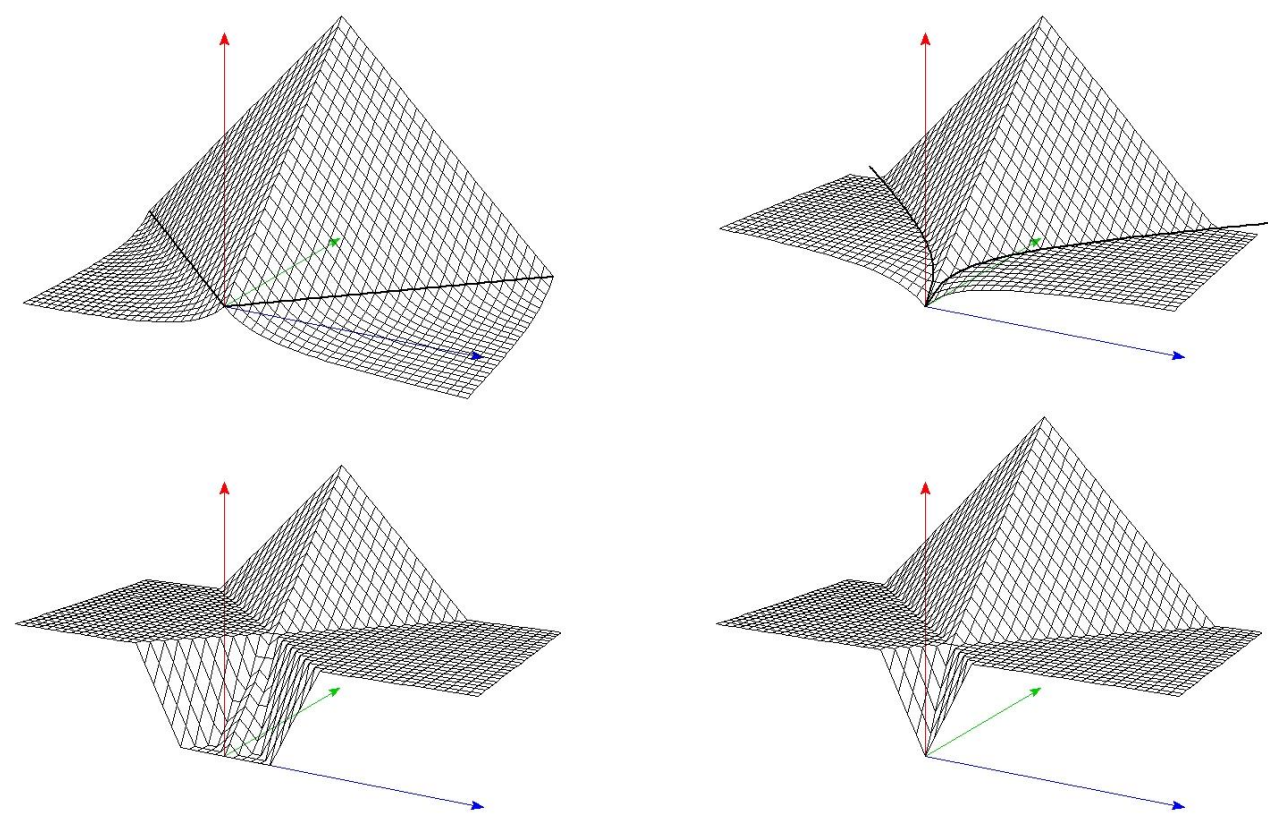

Figure 10: The envelope solutions of $\partial_{t} u-|\nabla u|=I(x)$ under several initial data $u_{0}$. The upper left is the case that $u_{0}(x)=-|x| /(1+|x|)$ and the solid curve is $(x, t, u)=(s,|s|, 0)$. The upper right is the case that $u_{0}(x)=|x| /(1+|x|)$ and the solid curve is $\left(s, \sqrt{s^{2}+2|s|}, \sqrt{s^{2}+2|s|}-s\right)$. The lower left is the case that $u_{0}(x)=2 \min \left\{(|x|-1)_{+}, 1\right\}$. The lower right is the case that $u_{0}(x)=2 \min \{|x|, 1\}$. Each function is the unique envelope solution.

$0 \leqq t-|x| \leqq R$ and it is smaller than $b(|x|+t)$, and also $V(x, t)=c(t-|x|-R)+b(R)(s=t-|x|-R)$ for $t-|x| \geqq R$ and it is bigger than $b(|x|+t)=b(R)$. Thus we conclude that

$$
v(x, t)= \begin{cases}b(|x|+t) & (t \leqq|x|+R), \\ c(t-|x|-R)+b(R) & (t \geqq|x|+R) .\end{cases}
$$

It is seen that $v(x, t) \equiv b(R)$ if $-|x|+R \leqq t \leqq|x|+R$. In this case, there is no effect of the step source by time $R$ on account of rapid growth of the initial data and $v$ becomes flat at time $R$. The solution for $c=1$ and $b(\rho)=2 \min \{\rho, 1\}(R=1)$ is given in Figure 10 (the lower right).

We next consider the case that $H(x, p)=|p|-r(x)=-\min _{a \in \bar{B}_{1}(0)}\langle a, p\rangle-r(x)$ with $r \in B U S C\left(\mathbf{R}^{n}\right)$, which describes the isotropic shrink at a velocity of 1 . Then Remark 5.4 guarantees that $v$ defined by

$$
v(x, t):=\inf _{\alpha \in \mathcal{A}}\left\{\int_{0}^{t} r\left(X^{\alpha}(s)\right) d s+u_{0}\left(X^{\alpha}(t)\right)\right\}
$$

is the unique envelope solution.

Example 5.8. Let us consider the case of (1.11) with a general initial condition $u_{0} \in B U C\left(\mathbf{R}^{n}\right)$. In this case, since for each $x \in \mathbf{R}^{n}$ the optimal control forces the corresponding state to go to the minimizer of $u_{0}$ on $\bar{B}_{t}(x)$ (and not to stay each $a_{j}$ for a positive time), we have

$$
v(x, t)=\min _{\bar{B}_{t}(x)} u_{0}
$$

This coincides with the solution of $\partial_{t} u+|\nabla u|=0$, and hence we may think that there is no effect of the source term $r(x)$. 
Example 5.9. Let us consider the case of (1.13) and $u_{0} \equiv 0$. In this case, since for each $x \in \mathbf{R}^{n}$ the optimal control forces the corresponding state to leave $S$ for the shortest time and stay in the outside of $S$ after the exit, we have

$$
v(x, t)=c \cdot \min \left\{t, \operatorname{dist}\left(x, S^{c}\right)\right\},
$$

where $S^{c}$ means a complementary set of $S$ in $\mathbf{R}^{n}$. In particular, if $S$ has no interior point, we see $v(x, t)=0$, which reduces to a special case of Example 5.8. We also learn for a bounded $S$ that $v(x, t)=c \cdot \operatorname{dist}\left(x, S^{c}\right)$ for every $t \geqq \sup _{x \in \mathbf{R}^{n}} \operatorname{dist}\left(x, S^{c}\right) \neq \infty$. The solution in the case that $S=[-1,1]$ and $c=1$ is given in Figure 11 .

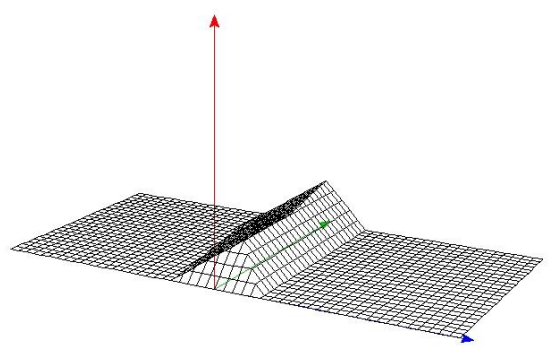

Figure 11: The envelope solution of $\partial_{t} u+|\nabla u|=\chi_{[-1,1]}(x), u_{0} \equiv 0$.

\subsection{Solutions without coercivity assumption}

In this subsection we focus on the equations of the form

$$
\text { (HJ0) } \begin{cases}\partial_{t} u-H_{1}(\nabla u)=c I(x) & \text { in } Q, \\ \left.u\right|_{t=0} \equiv 0 & \text { in } \mathbf{R}^{n},\end{cases}
$$

i.e., $H(x, p)=-H_{1}(p)-c I(x)$ with $H_{1}: \mathbf{R}^{n} \rightarrow \mathbf{R}$ and $c>0$. We do not impose the coercivity assumption on $H_{1}$ here. Also, without loss of generality we may take

$$
H_{1}(0)=0 ;
$$

if not, we replace $H_{1}(\nabla u)$ in $(5.9)$ with $H_{1}(\nabla u)-H_{1}(0)$ and solve the new (HJ0). For any solution $u(x, t)$ of the new one, $u(x, t)+H_{1}(0) t$ is a solution of the original (HJ0).

The next proposition helps us to construct envelope supersolutions when the step source consists of a singleton.

Proposition 5.10 (construction of envelope supersolutions). Assume (5.11). Assume that $u: Q \rightarrow \mathbf{R}$ is bounded from below and satisfies the following three conditions.

(i) $\tau+H(\hat{x}, p) \geqq 0$ for all $(\hat{x}, \hat{t}) \in\left(\mathbf{R}^{n} \backslash\{0\}\right) \times(0, T)$ and $(p, \tau) \in \bar{D}^{-} u_{*}(\hat{x}, \hat{t})$.

(ii) $u$ is continuous on $\{0\} \times(0, T)$.

(iii) $u(0, t)=$ ct in $(0, T)$ and $u(x, t) \leqq c t$ in $Q$.

Then $u \in \operatorname{e.SUP}(H)$.

Set $Q_{*}:=\left(\mathbf{R}^{n} \backslash\{0\}\right) \times(0, T)$. We say that $u$ is a $\bar{D}$-viscosity supersolution in $Q_{*}($ write $u \in \bar{D}-S U P(H)$ in $Q_{*}$ ) if $u$ satisfies the condition (i) and is bounded from below. 
Proof. For $\varepsilon>0$ we define

$$
u^{\varepsilon}(x, t):=\min \{u(x, t)+\varepsilon t, c t\} .
$$

(See Figure 12.) Then, we deduce by Proposition 3.5 that $u^{\varepsilon} \in \bar{D}-S U P(H)$ since we have the following three facts.

- $u(x, t)+\varepsilon t \in \bar{D}-S U P(H)$ in $Q_{*}$.

- $c t \in \bar{D}-S U P(H)$.

- $u(x, t)+\varepsilon t>c t$ in some open neighborhood of $(0, \hat{t}) \in Q$, where $\hat{t} \in(0, T)$.

Also, it is clear that $u=\inf _{\varepsilon>0} u^{\varepsilon}$. We thus conclude that $u \in e . S U P(H)$.

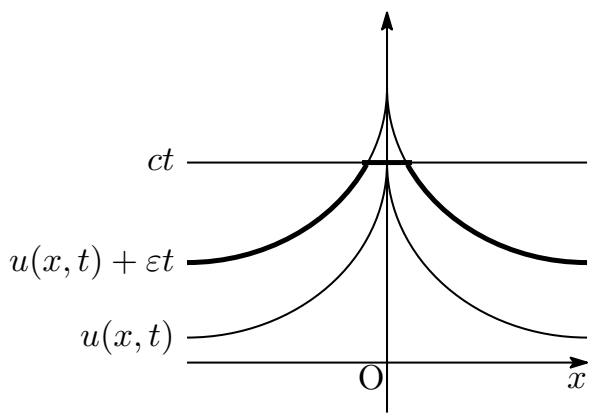

Figure 12: The definition of $u^{\varepsilon}$.

Let $a, b>0$ and define a "cone-shaped" function $W_{a, b}(x, t):=(a t-b|x|)_{+}$. Before describing the first existence result of (HJ0), which claims that $W_{c, b}$ is an envelope solution for suitable $b$ and $H_{1}$, we give simple observations about sub- and superdifferentials of the cone-shaped functions.

(CS) Let $\left(p_{+}, \tau_{+}\right) \in D^{+} W_{a, b}(\hat{x}, \hat{t})$ and $\left(p_{-}, \tau_{-}\right) \in D^{-} W_{a, b}(\hat{x}, \hat{t})$ for $(\hat{x}, \hat{t}) \in Q$.

(1) If $a \hat{t}<b|\hat{x}|$, we have $p_{ \pm}=0$ and $\tau_{ \pm}=0$.

(2) If $a \hat{t}>b|\hat{x}|$ and $\hat{x} \neq 0$, we have $\left|p_{ \pm}\right|=b$ and $\tau_{ \pm}=a$.

(3) If $a \hat{t}=b|\hat{x}|$, we have $\left|p_{-}\right| \leqq b, 0 \leqq \tau_{-} \leqq a$ and $b \tau_{-}=a\left|p_{-}\right|$. Also, $D^{+} W_{a, b}(\hat{x}, \hat{t})=\emptyset$.

(4) If $\hat{x}=0$, we have $\left|p_{+}\right| \leqq b$ and $\tau_{+}=a$. Also, $D^{-} W_{a, b}(\hat{x}, \hat{t})=\emptyset$.

Proposition 5.11. Assume (5.11) and let $b>0$. Assume that $H_{1}$ satisfies the following.

$$
\begin{gathered}
0 \leqq H_{1}(p) \leqq \frac{c}{b}|p| \quad \text { for all } p \in \mathbf{R}^{n} \text { such that }|p| \leqq b . \\
H_{1}(p)=c \quad \text { for all } p \in \mathbf{R}^{n} \text { such that }|p|=b .
\end{gathered}
$$

Then $W_{c, b}(x, t)=(c t-b|x|)_{+}$is an envelope solution of $(\mathrm{HJ} 0)$.

Proof. In view of $(5.12),(5.13)$ and (CS) we see that $W_{c, b}$ is a $\bar{D}$-subsolution of (5.9) and is a $\bar{D}$ supersolution of (5.9) in $Q_{*}$. Since $W_{c, b}$ fulfills the assumptions in Proposition 5.10, we conclude that $W_{c, b}$ is an envelope solution of (HJ0).

Of course, if $H_{1}$ fulfills the regularity assumption $\left(\mathrm{H}_{p}\right)$ required in our comparison principles, it follows that the Lipschitz continuous function $W_{c, b}$ is a unique envelope solution.

Example 5.12. We consider the case that $H_{1}(p)=|p|^{\alpha}$ with $\alpha>1$. Then, the conditions (5.12) and (5.13) are satisfied if we take $b=c^{1 / \alpha}$. Hence we see that $W_{c, b}(x, t)=\left(c t-c^{1 / \alpha}|x|\right)+$ is an envelope solution of (HJ0). 
Example 5.13. We next consider the case that $H_{1}(p)=\sqrt{1+|p|^{2}}-1$. Then, the conditions (5.12) and (5.13) are satisfied if we take $b=\sqrt{c^{2}+2 c}$. Hence we see that $W_{c, b}(x, t)=\left(c t-\sqrt{c^{2}+2 c}|x|\right)+$ is a unique envelope solution of (HJ0). In other words, the function $u(x, t)=\left(c t-\sqrt{c^{2}+2 c}|x|\right)_{+}+t$ is a unique envelope solution of

$$
\begin{cases}\partial_{t} u-\sqrt{1+|\nabla u|^{2}}=c I(x) & \text { in } Q \\ \left.u\right|_{t=0} \equiv 0 & \text { in } \mathbf{R}^{n}\end{cases}
$$

Unfortunately, Proposition 5.11 does not include the case that $H_{1}$ is "spokewisely concave" from the origin, that is, the case when

$$
H_{1}(p)=h(|p|), \quad \text { where } h:[0, \infty) \rightarrow \mathbf{R} \text { is strictly concave. }
$$

Here we say $h$ is strictly concave if $h((1-\lambda) x+\lambda y)>(1-\lambda) h(x)+\lambda h(y)$ for every $\lambda \in(0,1)$ and $x, y \in[0, \infty)$ with $x \neq y$. For the purpose of finding envelope solutions of (HJ0) in such cases we further assume that

$$
h \in C^{2}(0, \infty) \cap C[0, \infty), \quad h \text { is strictly increasing on }[0, \infty) .
$$

Then, it is easily seen by (CS) that

$$
W_{a, h^{-1}(a)}(x, t)=\left(a t-h^{-1}(a)|x|\right)_{+}
$$

is a $\bar{D}$-subsolution of (HJ0) for each $a \in(0, c)$ with $c \leqq\|h\|_{[0, \infty)}$. Besides, it turns out that these supremum

$$
U_{c}(x, t)=\sup _{a \in(0, c)}\left(a t-h^{-1}(a)|x|\right) \quad(c \leqq\|h\|)
$$

becomes an envelope solution of (HJ0).

Proposition 5.14. Assume (5.11), (5.14) and (5.15).

(1) Assume $c<\|h\|$. Then $U_{c}$ is a unique envelope solution of (HJ0).

(2) Assume $c=\|h\|$. Then $U_{\|h\|}$ is a $\bar{D}$-solution of (HJ0) and a unique envelope solution.

(3) Assume $c>\|h\|$. Then $U_{\|h\|}+k t I(x)$ are $\bar{D}$-solutions of (HJ0) for all $k \in[0, c-\|h\|]$.

We remark that the assumption $\left(\mathrm{H}_{p}\right)$ is satisfied because of the concavity of $h$; indeed, we now have $\left|H_{1}(p)-H_{1}(q)\right| \leqq h(|p-q|)$. Hence the uniqueness in (1) follows from the Lipschitz continuity of $U_{c}$.

Proof. At first, it is obvious that $U_{c}$ is a standard subsolution due to the stability under supremum. In order to prove that $U_{c}$ is an envelope supersolution we utilize Proposition 5.10. Notice that $U_{c}$ is rewritten as

$$
U_{c}(x, t)= \begin{cases}c t-b|x| & \left(|x| \leqq h^{\prime}(b) t\right) \\ h\left(g\left(\frac{|x|}{t}\right)\right) t-g\left(\frac{|x|}{t}\right)|x| & \left(h^{\prime}(b) t<|x|<h^{\prime}(0) t\right) \\ 0 & \left(h^{\prime}(0) t \leqq|x|\right)\end{cases}
$$

by a direct calculation. Here $h^{\prime}(0)$ means the right derivative at 0 and possibly equals $+\infty$. Also, we write $b=h^{-1}(c)$ and $g=\left(h^{\prime}\right)^{-1}$ for the inverse function of $h^{\prime}$. If $c=\|h\|$, we read (5.16) as

$$
U_{c}(x, t)= \begin{cases}c t & (x=0), \\ h\left(g\left(\frac{|x|}{t}\right)\right) t-g\left(\frac{|x|}{t}\right)|x| & \left(0<|x|<h^{\prime}(0) t\right), \\ 0 & \left(h^{\prime}(0) t \leqq|x|\right) .\end{cases}
$$


By the formula (5.16) we find $U_{c} \in C^{1}\left(Q_{*}\right)$ and its derivatives are as follows:

$$
\begin{aligned}
& \partial_{t} U_{c}(x, t)= \begin{cases}c & \left(|x| \leqq h^{\prime}(b) t\right), \\
h\left(g\left(\frac{|x|}{t}\right)\right) & \left(h^{\prime}(b) t<|x|<h^{\prime}(0) t\right), \\
0 & \left(h^{\prime}(0) t \leqq|x|\right) .\end{cases} \\
& \nabla U_{c}(x, t)= \begin{cases}-b \frac{x}{|x|} & \left(0<|x| \leqq h^{\prime}(b) t\right), \\
-g\left(\frac{|x|}{t}\right) \frac{x}{|x|} & \left(h^{\prime}(b) t<|x|<h^{\prime}(0) t\right), \\
0 & \left(h^{\prime}(0) t \leqq|x|\right) .\end{cases}
\end{aligned}
$$

Thus we deduce that

$$
\partial_{t} U_{c}(x, t)-h\left(\left|\nabla U_{c}(x, t)\right|\right)=0 \quad \text { for all }(x, t) \in Q_{*},
$$

and hence $U_{c}$ satisfies the condition (i) in Proposition 5.10. Since the conditions (ii) and (iii) are clear, we conclude that $U_{c}$ is an envelope supersolution. If $c=\|h\|$ in particular, we see that $U_{c}$ is also a $\bar{D}$-supersolution of (HJ0) since $\bar{D}^{-} U_{c}(0, t)=\emptyset$.

(2) To show the uniqueness of $U_{c}$ as an envelope solution we use the idea of Remark 3.14. It is seen that $U_{c}$ is the minimal envelope solution because $U_{c}=\sup _{a \in(0, c)} W_{a, h^{-1}(a)}$ and $W_{a, h^{-1}(a)} \in$ $\operatorname{SUB}(H, 0) \cap B L i p(Q)$. Next, let $u^{\varepsilon}(x, t):=\min \left\{U_{c}(x, t)+\varepsilon t, c t\right\}$ for $\varepsilon>0$. As we showed in the proof of Proposition 5.10, it turns out that $u^{\varepsilon} \in \bar{D}-S U P(H)$. We also have $u^{\varepsilon} \in B L i p(Q)$ and $U_{c}=\inf _{\varepsilon>0} u^{\varepsilon}$, and therefore $U_{c}$ is the maximal envelope solution.

(3) This claim follows from (2) and the fact that $\left(U_{\|h\|}+k t I(x)\right)_{*}=U_{\|h\|}$.

Example 5.15. We consider the case that $H_{1}(p)=|p| /(1+|p|)$, which is a non-coercive Hamiltonian. Then, by substituting

$$
b=\frac{c}{1-c}, \quad h(r)=\frac{r}{1+r}, \quad g(r)=\frac{1-\sqrt{r}}{\sqrt{r}}
$$

into (5.16) we see that

$$
U_{c}(x, t)= \begin{cases}c t-\frac{c}{1-c}|x| & \left(|x| \leqq(1-c)^{2} t\right), \\ \left\{(\sqrt{t}-\sqrt{|x|})_{+}\right\}^{2} & \left(|x| \geqq(1-c)^{2} t\right)\end{cases}
$$

is a unique envelope solution of (HJ0) when $c \leqq 1=\|h\|$, and in particular $U_{1}(x, t)=\left\{(\sqrt{t}-\sqrt{|x|})_{+}\right\}^{2}$ is a $\bar{D}$-solution of (HJ0) with $c=1$. In the case when $c>1$, functions $U_{1}(x, t)+k t I(x)$ are all $\bar{D}$-solutions of (HJ0) for $k \in[0, c-1]$. (See Figure 13.) Also, we see that the Lipschitz continuity preserving property breaks down for $c \geqq 1$ since $U_{1}$ is not Lipschitz continuous in $Q$.

Example 5.16. We next consider the case that $H_{1}(p)=|p|^{\alpha}$ with $0<\alpha<1$. Then, by substituting

$$
b=c^{1 / \alpha}, \quad h(r)=r^{\alpha}, \quad g(r)=\left(\frac{\alpha}{r}\right)^{1 /(1-\alpha)}
$$

into (5.16) we see that

$$
U_{c}(x, t)= \begin{cases}c t-c^{1 / \alpha}|x| & \left(|x| \leqq \alpha t / c^{(1-\alpha) / \alpha}\right), \\ \left(\frac{\alpha t}{|x|}\right)^{\alpha /(1-\alpha)} t-\left(\frac{\alpha t}{|x|}\right)^{1 /(1-\alpha)}|x| & \left(|x| \geqq \alpha t / c^{(1-\alpha) / \alpha}\right)\end{cases}
$$

is a unique envelope solution of (HJ0) for any $c>0$. This formula means that the present equation (HJ0) has a some kind of infinite propagation property for a step source because $u>0$ in $Q$. (See Figure 14.)

The formula (5.16) also applies to Hamiltonians with the hyperbolic tangent form ([26])

$$
H(x, p)=-|p| \tanh \frac{1}{|p|}-c I(x),
$$

but it is complicated to calculate the inverse function $g=\left(h^{\prime}\right)^{-1}$ in this case. 

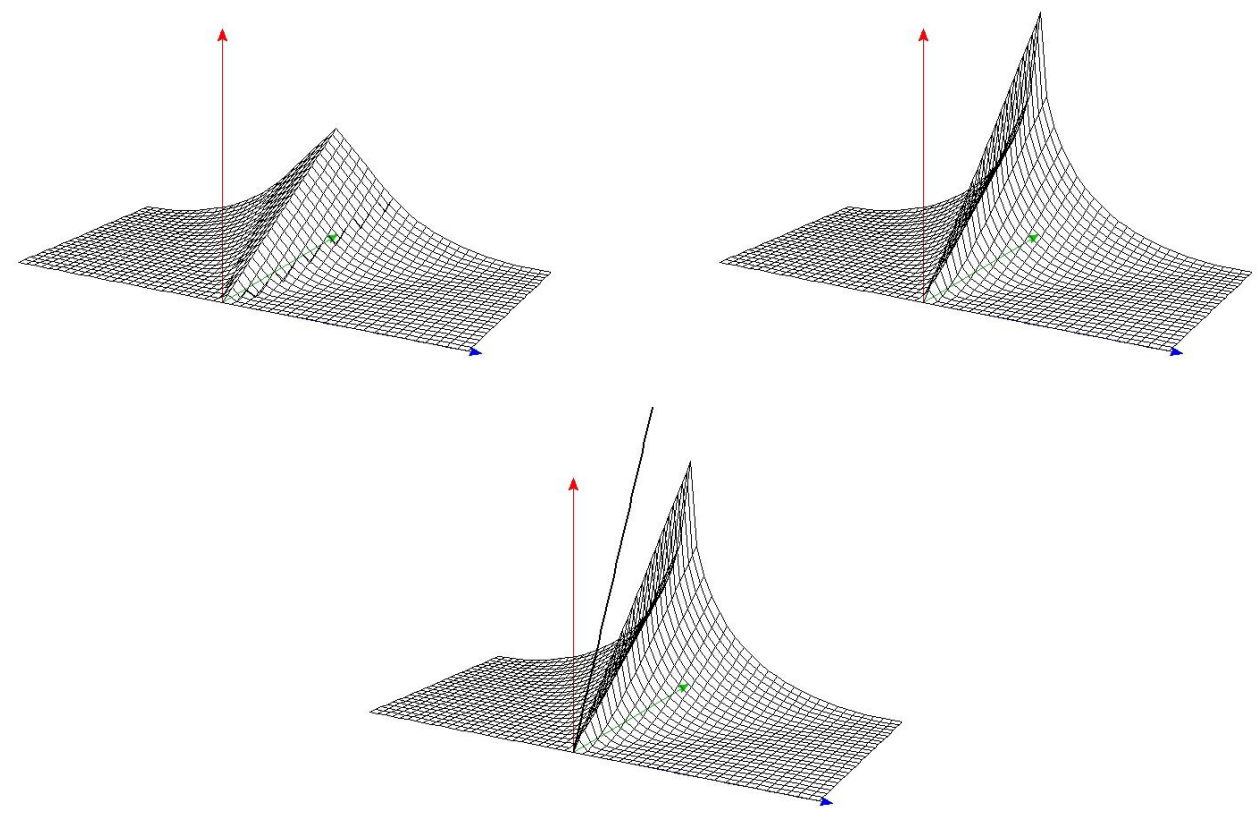

Figure 13: Solutions of $\partial_{t} u-|\nabla u| /(1+|\nabla u|)=c I(x), u_{0} \equiv 0$. The envelope solution for $c=1 / 2$ (the upper left), the $\bar{D}$-solution for $c=1$ (the upper right) and one of the $\bar{D}$-solutions for $c=2$ (the bottom).

\subsection{Remark on relation to Dirichlet boundary problems}

Let $u$ be the unique envelope solution of the problem with a single step source:

$$
\begin{cases}\partial_{t} u-|\nabla u|=c I(x) & \text { in } \mathbf{R}^{n} \times(0, T), \\ u(x, 0)=u_{0}(x) & \text { in } \mathbf{R}^{n} .\end{cases}
$$

We study in this subsection whether $u$ is also a solution of the Dirichlet boundary problem:

$$
\text { (Di) } \begin{cases}\partial_{t} u-|\nabla u|=0 & \text { in }\left(\mathbf{R}^{n} \backslash\{0\}\right) \times(0, T), \\ u(x, 0)=u_{0}(x) & \text { in } \mathbf{R}^{n}, \\ u(0, t)=c t & \text { in }(0, T) .\end{cases}
$$

To simplify the argument we assume $u_{0}(0)=0$. We first recall the following facts about $u$ from the observation in Example 5.7.

(1) $u(0, t) \geqq c t$ for all $t \in(0, T)$.

(2) $u(0, t)=c t$ for all $t \in(0, T)$ provided that $u_{0}(x) \leqq c|x|$ in $\mathbf{R}^{n}$.

(3) If

$$
u_{0}(x)=2 c \min \{|x|, 1\},
$$

whose slope is larger than $c$ near the origin, then the unique envelope solution is

$$
v(x, t)= \begin{cases}2 c \min \{|x|+t, 1\} & (t \leqq 1), \\ c(t-|x|-1)_{+}+2 c & (t \geqq 1) .\end{cases}
$$

In particular, we have $v(0, t)=\min \{2 c t, c(t+1)\}>c t$. 


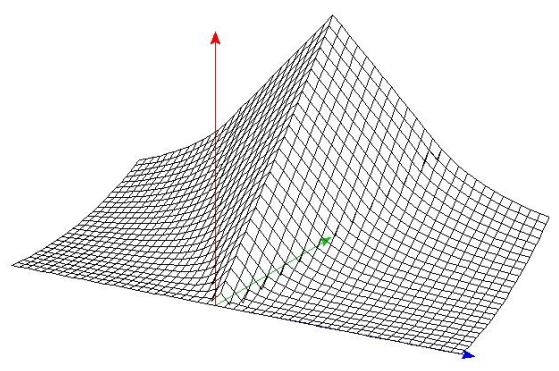

Figure 14: The envelope solution of $\partial_{t} u-\sqrt{|\nabla u|}=I(x), u_{0} \equiv 0$.

We see by (1) that $u$ is always a supersolution of (Di). Also, by virtue of (2), if $u_{0}(x) \leqq c|x|$ in $\mathbf{R}^{n}$, then $u$ is a viscosity solution of (Di) which indeed attains the boundary condition. What happens in the case that $u_{0}(x) \not c|x|$ in $\mathbf{R}^{n}$ ? Unfortunately, we cannot expect that $u$ is a subsolution on the boundary even in the weak sense, i.e., $u(0, t) \leqq c t$ or $\tau-|p| \leqq 0$ whenever $(p, \tau) \in D_{\mathbf{R}^{n}}^{+} u(0, t)$. In fact, when the initial data is given by (5.18), we have $v(0,2)>2 c$ and $\tau-|p|>0$ for $(p, \tau)=(0, c) \in D^{+} v(0,2)$. Instead of $v$, if we set

$$
v^{\tau}(x, t)= \begin{cases}v(x, t) & (t \leqq 1), \\ 2 c & (1 \leqq t \leqq 1+\tau), \\ v(x, t-\tau) & (t \geqq 1+\tau),\end{cases}
$$

then each $v^{\tau}(\tau \geqq 1)$ becomes a solution of (Di) with (5.18) in the weak sense. One can interpret the constant $\tau$ as a "waiting time". The Dirichlet problem (Di) forces its solution to stop the growth until it satisfies the Dirichlet boundary data at the origin.

As an another type of the boundary condition in the weak sense, one may think of the dynamic boundary condition ([13]); namely

$$
\text { (Dy) } \begin{cases}\partial_{t} u-|\nabla u|=0 & \text { in }\left(\mathbf{R}^{n} \backslash\{0\}\right) \times(0, T), \\ u(x, 0)=u_{0}(x) & \text { in } \mathbf{R}^{n}, \\ \partial_{t} u(0, t)=c & \text { in }(0, T) .\end{cases}
$$

However, one cannot expect the uniqueness of solutions for (Dy) as well. Indeed, each $v^{\tau}(\tau \geqq 0)$ is a solution of (Dy) with (5.18).

\section{Acknowledgments}

The authors are grateful to Professor Etsuro Yokoyama for introducing them several important references on crystal growth. The authors are also grateful to Dr. Qing Liu for many helpful discussions especially on the optimal control theory.

The work of the first author is partly suppoted by Grant-in-aid for Scientific Research, No. 21224001 (Kiban S), the Japan Society for the Promotion of Science (JSPS). The work of the second author is suppoted by Grant-in-aid for Scientific Research of JSPS Fellows No. 23-4365.

\section{References}

[1] M. Bardi, S. Bottacin, On the Dirichlet problem for nonlinear degenerate elliptic equations and applications to optimal control, Rend. Sem. Mat. Univ. Politec. Torino 56 (1998), no. 4, 13-39. 
[2] M. Bardi, I. Capuzzo-Dolcetta, Optimal control and viscosity solutions of Hamilton-Jacobi-Bellman equations, With appendices by Maurizio Falcone and Pierpaolo Soravia, Systems \& Control: Foundations \& Applications, Birkhäuser Boston, Inc., Boston, MA, 1997.

[3] E. N. Barron, R. Jensen, Semicontinuous viscosity solutions for Hamilton-Jacobi equations with convex Hamiltonians, Comm. Partial Differential Equations 15 (1990), no. 12, 1713-1742.

[4] A. Briani, A. Davini, Monge solutions for discontinuous Hamiltonians, ESAIM Control Optim. Calc. Var. 11 (2005), no. 2, 229-251.

[5] W. K. Burton, N. Cabrera, F. C. Frank, The growth of crystals and the equilibrium structure of their surfaces, Philos. Trans. Roy. Soc. London. Ser. A. 243 (1951), 299-358.

[6] L. A. Caffarelli, M. G. Crandall, M. Kocan, A. Swięch, On viscosity solutions of fully nonlinear equations with measurable ingredients, Comm. Pure Appl. Math. 49 (1996), no. 4, 365-397.

[7] F. Camilli, A. Siconolfi, Time-dependent measurable Hamilton-Jacobi equations, Comm. Partial Differential Equations 30 (2005), no. 4-6, 813-847.

[8] X. Chen, B. Hu, Viscosity solutions of discontinuous Hamilton-Jacobi equations, Interfaces Free Bound. 10 (2008), no. 3, 339-359.

[9] M. G. Crandall, H. Ishii, P. L. Lions, User's guide to viscosity solutions of second order partial differential equations, Bull. Amer. Math. Soc. (N.S.) 27 (1992), no. 1, 1-67.

[10] K. Deckelnick, C. M. Elliott, Uniqueness and error analysis for Hamilton-Jacobi equations with discontinuities, Interfaces Free Bound. 6 (2004), no. 3, 329-349.

[11] K. Deckelnick, C. M. Elliott, Propagation of graphs in two-dimensional inhomogeneous media, Appl. Numer. Math. 56 (2006), no. 9, 1163-1178.

[12] C. De Zan, P. Soravia, Cauchy problems for noncoercive Hamilton-Jacobi-Issacs equations with discontinuous coefficients, Interfaces Free Bound. 12 (2010), no. 3, 347-368.

[13] C. M. Elliott, Y. Giga, S. Goto, Dynamic boundary conditions for Hamilton-Jacobi equations, SIAM J. Math. Anal. 34 (2003), no. 4, 861-881.

[14] L. C. Evans, Partial differential equations. Second edition, Graduate Studies in Mathematics, 19. American Mathematical Society, Providence, RI, 2010, xxii+749 pp.

[15] Y. Giga, Surface evolution equations: A level set approach, Monographs in Mathematics, 99, Birkhauser Verlag, Basel, 2006, xii+264 pp.

[16] Y. Giga, P. Górka, P. Rybka, A comparison principle for Hamilton-Jacobi equations with discontinuous Hamiltonians, Proc. Amer. Math. Soc. 139 (2011), no. 5, 1777-1785.

[17] Y. Giga, Q. Liu, H. Mitake, Large-time asymptotics for one-dimensional Dirichlet problems for Hamilton-Jacobi equations with noncoercive Hamiltonians, J. Differential Equations, to appear.

[18] H. Ishii, Hamilton-Jacobi equations with discontinuous Hamiltonians on arbitrary open sets, Bull. Fac. Sci. Engrg. Chuo Univ. 28 (1985), 33-77.

[19] S. A. Molchanov, D. Surgailis, W. A. Woyczynski, The large-scale structure of the universe and quasi-Voronoi tessellation of shock fronts in forced Burgers turbulence in $\mathbf{R}^{d}$, Ann. Appl. Probab. 7 (1997), no. 1, 200-228.

[20] R. T. Newcomb II, J. Su, Eikonal equations with discontinuities. Differential Integral Equations 8 (1995), no. 8, 1947-1960.

[21] N. Ostrov, Extending viscosity solutions to eikonal equations with discontinuous spatial dependence, Nonlinear Anal. 42 (2000), no. 4, Ser. A: Theory Methods, 709-736. 
[22] G. Sazaki, S. Zepeda, S. Nakatsubo, E. Yokoyama, Y. Furukawa, Elementary steps at the surface of ice crystals visualized by advanced optical microscopy, Proc. Nat. Acad. Sci. USA. 107 (2010), no. $46,19702-19707$.

[23] T. P. Schulze, R. V. Kohn, A geometric model for coarsening during spiral-mode growth of thin films, Phys. D 132 (1999), no. 4, 520-542.

[24] P. Soravia, Boundary value problems for Hamilton-Jacobi equations with discontinuous Lagrangian, Indiana Univ. Math. J. 51 (2002), no. 2, 451-477.

[25] P. Soravia, Degenerate eikonal equations with discontinuous refraction index, ESAIM Control Optim. Calc. Var. 12 (2006), no. 2, 216-230.

[26] E. Yokoyama, Y. Giga, P. Rybka, A microscopic time scale approximation to the behavior of the local slope on the faceted surface under a nonuniformity in supersaturation, Phys. D 237 (2008), no. $22,2845-2855$. 